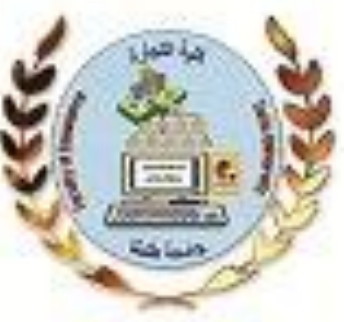

\title{
أثر الانفتاح التجاري علي التضخم: اختبار تحقق فرضية Romer
}

$$
\begin{aligned}
& \text { د . مـحمـد مسحمـد الـسيـد ر اضي } \\
& \text { مدرس بقسم الاقتصاد } \\
& \text { المعهة العالي للإدارة وتكنولوجيا المعلومات بكفر الثنيخ. }
\end{aligned}
$$

Mradi75@gmail.com

$$
\begin{aligned}
& \text { د .خـالــ إبـر اهـيـم سـيـد أحمـد } \\
& \text { أستاذ مساعد بقسم الاقتصاد و المالية العامة } \\
& \text { كلية التجارة-جامعة طنطا. }
\end{aligned}
$$

khaled.ahmed@commerce.tanta.edu.eg 
المستخلص: قام هذا البحث باختبار مدى تحقق فرضية Romer و التي تتص علي وجود علاقة عكسية بين الانفتاح التجاري و التضخم في الاقتصاد المصري .وتم ذلك باستخدام بيانات

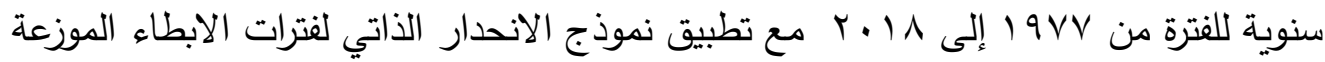
(ARDL)

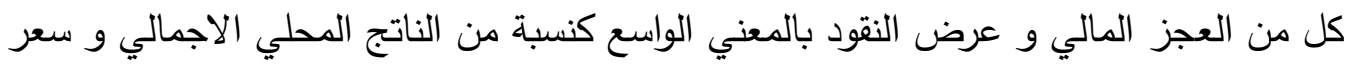
الصرف الرسمي للعملة المحلية كمتغيرات تحكم. كما تم التعبير عن الانفتاح التجاري باستخدام

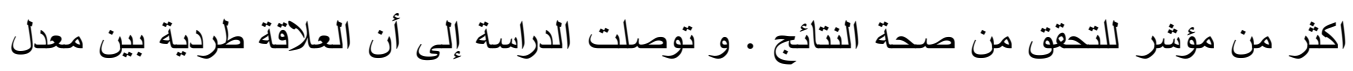

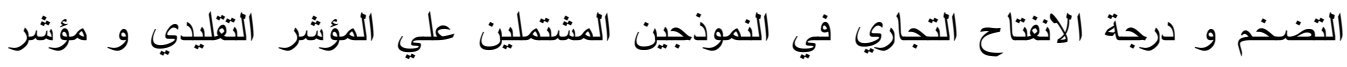
الواردات و عدم معنوية النموذج الثالث الذي يحتوي علي مؤشر العولمة كدليل على الانفتاح التجاري بمعناه الواسع مما يعني عدم تحقق فرضيةRomer (1993) في الاقتصاد المصري.

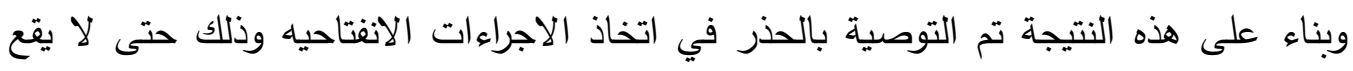
الاقتصاد في مشاكل ناتجة عن التضخم المستورد. الكلمات المفتاحية: الانفتاح التجاري و التضخم، فرضية(1993) Romer، الانحدار الذاتي لفترات الابطاء الموزعة (ARDL) 


\begin{abstract}
This research examined the validity of Romer's hypothesis, which states that there is an inverse relationship between trade openness and inflation, in the Egyptian economy. An Autoregressive Distributed Lags (ARDL) model was applied using annual data for the period( 1977 to 2018). The growth rate in real per capita GDP, fiscal deficit and the broad money supply as a percentage of GDP and the official exchange rate was used as control variables. The trade openness was also expressed by using more than one indicator to validate the results. The study found that the relationship between the rate of inflation and the degree of trade openness is positive in the two models that include the traditional index and the import index, But the third model that contains the globalization index as a proxy for trade openness in a broad sense. It means that the Romer hypothesis (1993) is not valid in the Egyptian economy. Based on this result, caution has been recommended in taking openness Procedures so as not to get into problems of imported inflation.
\end{abstract}

Keywords: trade openness and inflation, Romer's hypothesis (1993), Autoregressive Distributed Slowdown (ARDL) 


\section{1 - 1مقدمة}

على الرغم مـن أن الاحتفاظ بمستوى معين مـن التضـخم قد يكون أمـرا مرغوبـا فيـه لتحفيز النمو الاقتصـادي (Gokal and Hanif, 2004)، الا أن ارتفاع معدلات التضـخم ينطوي على تكاليف و أعباء على النظام الاقتصادي والاجتماعي. فالتضخم هو مؤشر يرتبط بالرفاهية (Mansilla et al, 2020) ،حيث يؤدي إلى تآكل القوة الشرائية للمستهلكين و تخفيض مستويات الرفاهيـة للفئـات الاقل دخـلا في المجتمـع. كمـا يؤدي الحى تغيير الأسـعار النسبية للمنتجات ومدخلات الإنتاج ، و يحجب أفق التتبؤ ممـا ينعكس في إهدار الكثير من الموارد في معاملات ومضـاربات غير منتجة ، و لذلك يمكن اعتباره من جهة اخرى عائقا أمام النمو الاقتصادي. كما يترتب علي التضخم سيادة حالة من عدم التأكد مما قد يخل بأسس اتخاذ Krugman, القرارات الاقتصـادية الرشـيدة ويضـر بمصداقية معظم السياسـات الحكوميـة.

و عـادة يكون التضـخم المرتفع مصـحوبًا بمعدلات فائدة حقيقيـة شديدة التقلب (و قد تكون سالبة). و هو مما يؤثر بالسلب على حجم و معدل الادخار في المجتمع. و قد يؤدي تدخل الحكومات في محاولة منها لحماية شرائح معينة من السكان من التضخم باستخدام تدابير انتقائية للتحكم في الأسعار إلى تشويه عمل آلية الأسعار (Ashra, 2002).

كل هذا و غيره مـن المشكلات التي يثيرهـا ارتفـاع معدلات التضـخم ، بالإضـافة الى تثابكه في علاقات معقدة مسع الكثير من المتغيرات الاقتصـادية و الاجتماعية يدفع الى اعتبار تحقيق الاستقرار في الاسعار و المحافظة علي هذا الاستقرار هدفا رئيسيا للسياسـة الاقتصادية في معظم دول العـالم. ولقد تعددت النظريـات والابحـاث التي تدرس العوامـل التي تزيـــ مـن الضـوط التضخمية في الاقتصـاد وطرق تخفيفها. ولعل من الاسهامات التي أثارت الجدل في 
هذ المجال دراسة (1993) Romer و التي انتهى فيها الى أن الانفتاح التجاري يؤثر عكسيا على معدل التضخم و هو ما أطلق عليه " فرضية Romer's hypothesis" "Romer". وقد أرجع Romer سبب التأثير العكسي للانفتاح التجاري على التضخم إلى أن السياسة النقدية التوسعية غير المتوقعة تؤدي إلى انخفاض في سعر الصرف الحقيقي، واضرار الانخفاض في

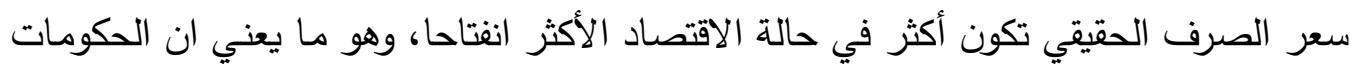
سوف تتجنب استخدام السياسات النقدية التوسعية كلما زادت درجة الانفتاح التجاري بها، وبالتالي تخفض فيها معدلات التضخم (Romer, 1993).

من الناحية النظريـة، يوجد رأيـان لتفسير العلاقة بين الانفتاح التجاري والتضـخ. الرأي الأول

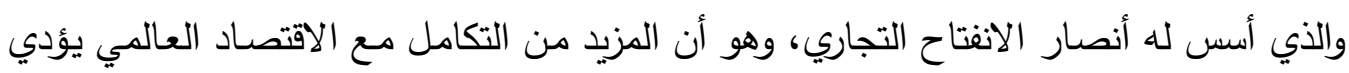
إلى انخفاض معدل التضـخم، حيث أن قنوات المنافسـة والإنتاجيـة هي التي تجعل الانفتـاح التجاري ذو تأثير على معدل التضخم من خلال زيادة المنافسة والتخصيص الكفه للموارد مما يحد من قدرة الشركات على التسعير للمنتجات وبالتالي ينخفض معدل التضخم في الاقتصاديات ذات الحجم الصغير في ظل الانفتاح التجاري . (Binici et al., 2012) وعلى العكس من الرأي السابق، تؤكد فرضية دفع التكاليف على أن الانفتاح التجاري يؤدي إلى

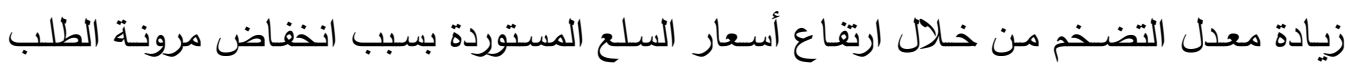
عليها (Evans, 2007)، ويمكن أن يحدث ارتفاع معدل التضخم نتيجة الانفتاح التجاري الناتج مرنج

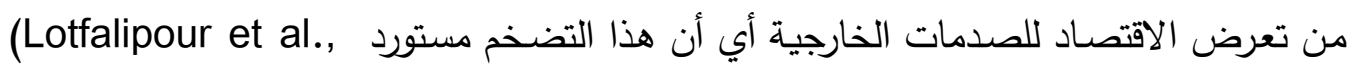
(2013. كمـا أن الانفتـاح التجـاري يحد مـن قدرة السلطات النقديـة في السيطرة على معدل التضخم (Zakaria, 2010). 
و مع تعدد الدراسات التي تمت لاختبار صحة فرضية Romer ، و مع تنوع الأساليب القياسية المستخدمة في دراستها، إلا أن نتائج هذه الدراسات التطبيقية لم تحسم الجدل. فقد اختلفت النتائج بين الدول و داخل نفس الدولة باختلاف الفترات الزمنية محل الدراسة.

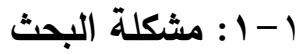

منذ السبعينات من القرن الماضي يتخذ الاقتصاد المصري خطوات واضحة نحو التحرر الاقتصادي و الانفتاح التجاري سعيا وراء تحقيق اقصى استفادة مكنة و التزاما بالاتفاقيات الدولية الموقعة بدءا من اتفاقيات الجات و وصولا الى منظمة التجارة العالمية. و يتضح ذلك

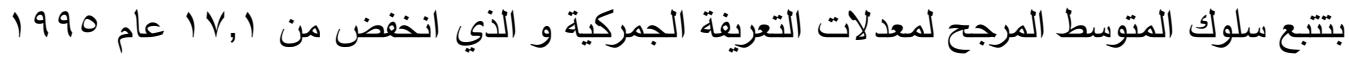

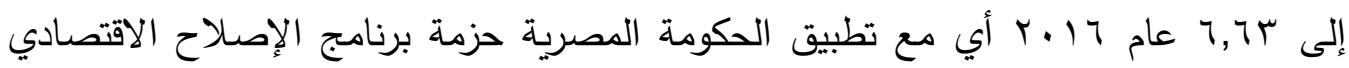

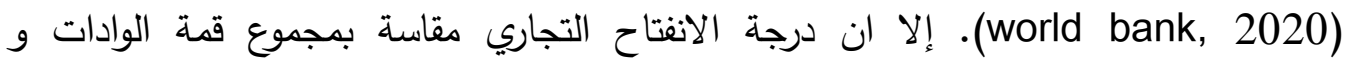
الصادرات من السلع و الخدمات الى الناتج المحلي الاجمالي خضعت للعديد من التقلبات خلال فترة الدراسة كما يتضح من الثكل التالي رقم (1). و يتزامن ذلك مع معاناة الاقتصاد المصري على فترات متفاوتة من الارتفاع الثديد في معدلات التضخم، و عادة ما تعقب هذه الفترات اتخاذ الحكومة لإجراءات مالية و نقدية متثددة لـكافحة هذا التضخم • و يتضح ذلك من التذبذب في معدلات التضخم الذي يوضحه الثكل رقم (1). 


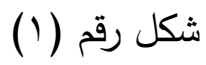

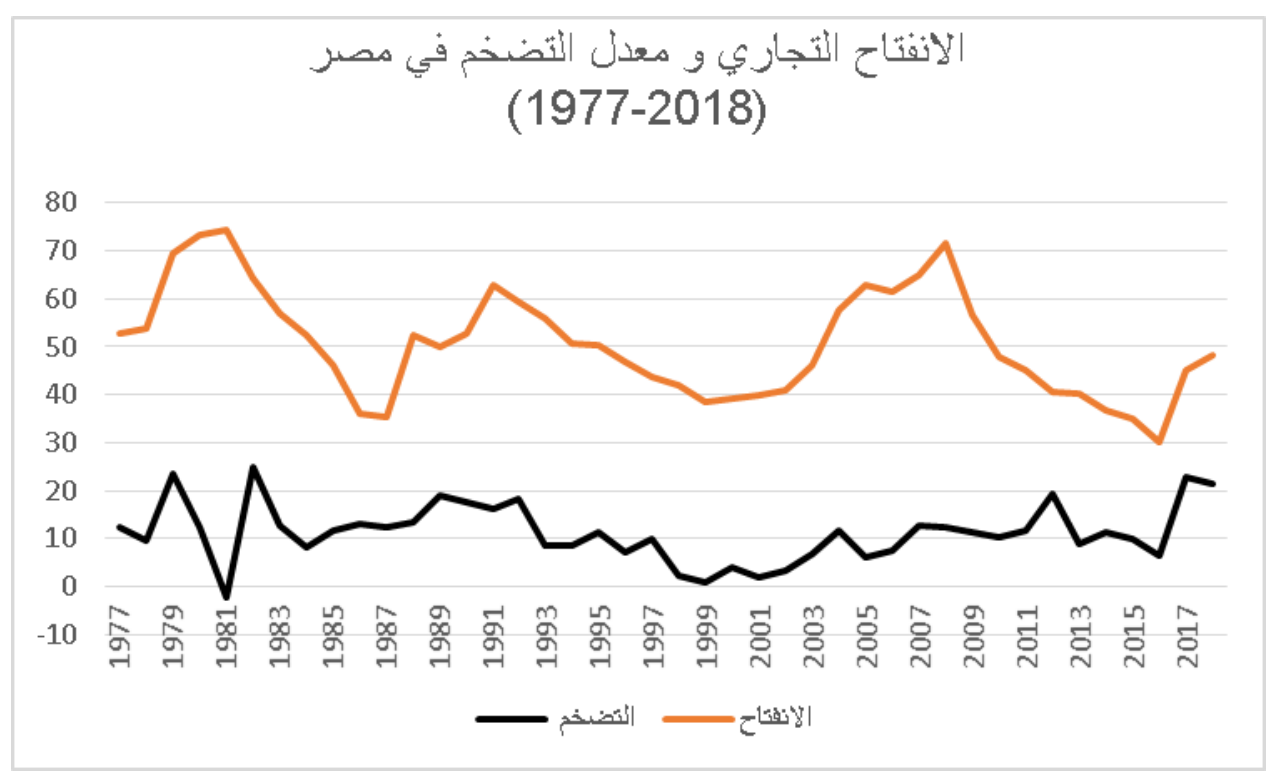

مصدر البيانات: مorld Development Indicators | DataBank

(worldbank.org)

ونظرا للمشكلات الهامة التي يشيرها ارتفاع معدلات التضخم وارتباط التضخم بعلاقات

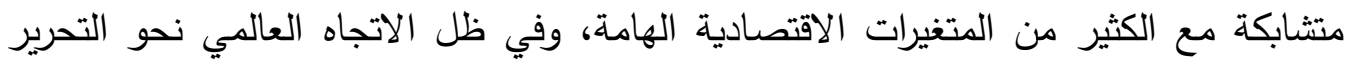

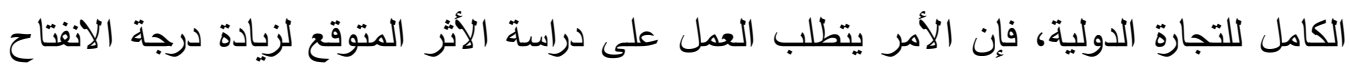
التجاري على معدل التضخم. ففي حال تحقق فرضية Romer في الاقتصاد المصري قد يكون

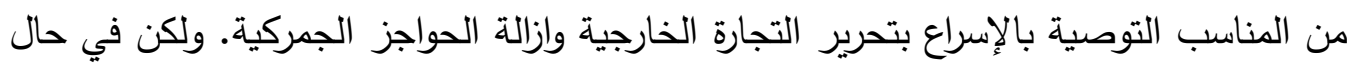

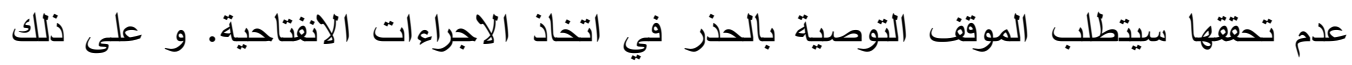

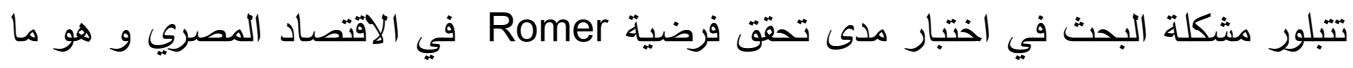

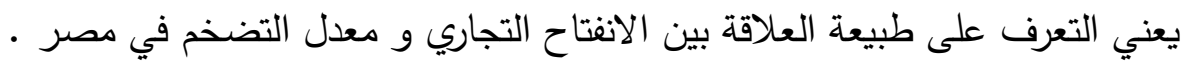




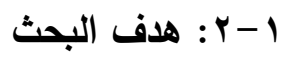

يستهد البحث اختبار مدي تحقق فرضية Romer في الاقتصاد المصري و هو ما

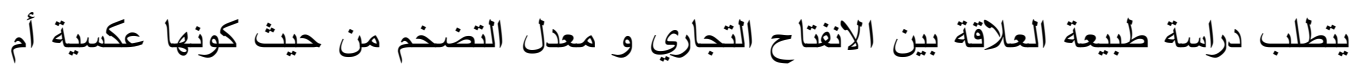

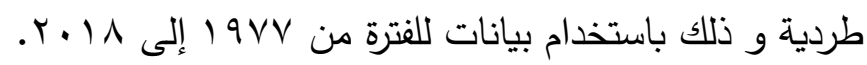

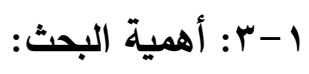

يستمد الموضوع اهيته من خطورة الظاهرة التي يتناولها، فالاختلاف في نتائج الأبحاث التي تتناول اختبار تحقق هذه الفرضية باختلاف الدول محل الدراسة يجعل من الضروري التحقق من تحققها في الاقتصاد المصري حتى نتجنب خطأ اتخاذ قرارات تتعلق بزيادة او الحد من الانفتاح التجاري و يكون لها اثار غير مرغوبة علي معدلات التضخم و غيره من المتغيرات الهامة

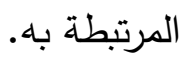

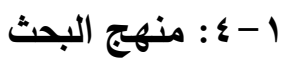

يستخدم البحث التحليل القياسي اعتمادا على تحليل بيانات السلاسل الزمنية لدرجة الانفتاح التجاري ومعدل التضخم بالإضافة إلى بعض المتغيرات الأخرى التي قد تؤثر على معدلات

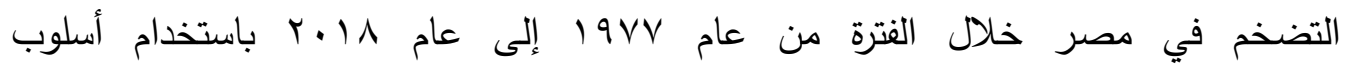
Autoregressive distributed lag (ARDL) القصير والطويل، وقد تم استخدام برنامج التحليل الإحصائي EViews لإجراء الاختبارات

ينقسم البحث إلى أربعة أقسام بالإضافة إلى المقدمة وهي على الترتيب الإطار النظري للبحث

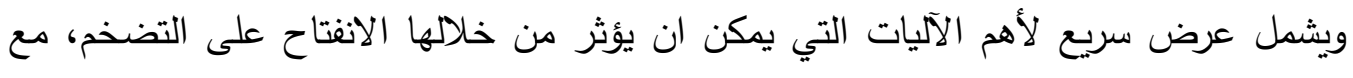


توضيح لفرضية Romer والآراء المعارضة لها. و يتم استعراض بعض الدراسات السابقة لطبيعة العلاقة بين الانفتاح التجاري و التضخم في القسم الثالث، ويتبع ذلك الدراسة التطبيقية في القسم الرابع للبحث و نختتم بالنتائج و التوصيات .

ب: ب الإطار النظري للبحث

يُعبر الانفتاح التجاري عن تخفيض التعريفة الجمركية بالإضافة إلى إزالة كافة الحواجز و العقبات امـام التجارة الدولية و عـادة مـا يقاس بنسبة مجموع الصـادرات والواردات إلى النـاتج المحلي الإجمالي أو نسبة الواردات فقط إلى الناتج المحلي الإجمالي. ومن الناحية النظريـة ،

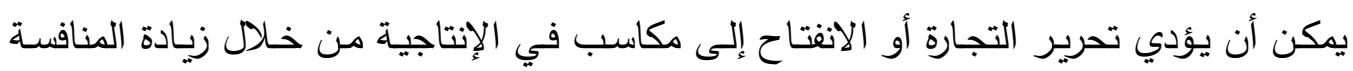

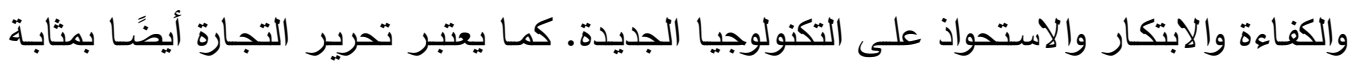
توسيع للفرص الاقتصادية من خلال توسيع حجم السوق وتعزيز تأثير انتشار المعرفة Sikdar) get al. 2013).

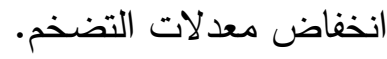
و لتوضيح الجدل النظري حول علاقة الانفتاح بالتضخم يمكن استعراض ما يلي: r-1:أهم آليات تأثير الانفتاح على التضخم

يمكن ان ينتج تأثر الانفتاح على التضخم من خلال تأثيره الإيجابي على الإنتاج والانتاجية في الاقتصاد، والذي من المرجح أن يخفف الضغط على الأسعار يمكن أن يتم ذلك من خلال الإل :(Ashra, 2002) - زيـادة الكفـاءة التي مـن المرجح أن تقلل من تكـاليف الانتـاج بإحداث تغييرات في تكوين المدخلات 
- ما قد ينجم عنه من تخصيص أفضل للموارد

- يمكن أن يزيد من الاستثمار الأجنبي الداخل، والذي يمكن أن يحفز نمو الإنتاج وبالتالي يخفق من الضغط عن مستوى السعر.

كما أن درجة تكامل الاقتصاد المحلي مع الاقتصاد العالمي يمكن أن يقلل من التقلبات السعرية المحلية الناتجة عن اي صدمات في الانتاج المحلي حيث يمكن معادلة هذه الصدمات من خلال تدفق الانتاج الي الداخل او الخارج. و كذلك يمكن أن تؤثر على مستوى الأسعار المحلية ، حيث من المرجح أن يستجيب المنتجون المحليون للأسعار الدولية وليس فقط لمستوى الأسعار المحلية. و قد يؤدي ذلك إلى ضغط لأعلي على أسعار السلع التي تُباع في الاقتصاد المحلي

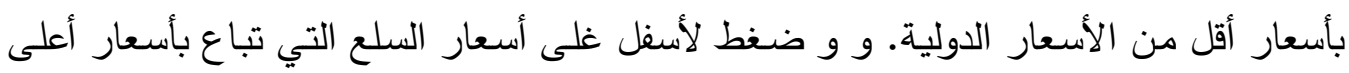

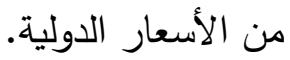

و مع التزام منظمة التجارة العالمية بتنسيق هيكل التعريفة الجمركية عبر البلدان ، من المرجح أن تنخفض تكلفة استيراد نسبة كبيرة من السلع المتداولية. ،و سيكون لهذا تأثير على معدل التضخم المحلي فقط في المرحلة الانتقالية.

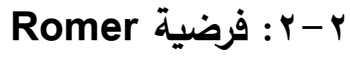

فكر ( Romer (199) في فرضبة وجود علاقة عكسية بين الانفتاح التجاري والتضخم، ولاختبار هذه الفرضية قام بإجراء انحدار لوغاريتم معدل التضخم على الانفتاح التجاري مقاسا

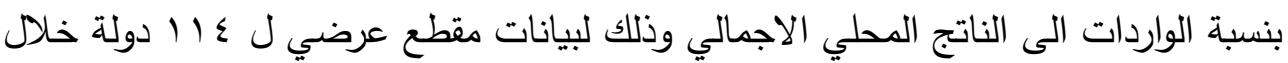

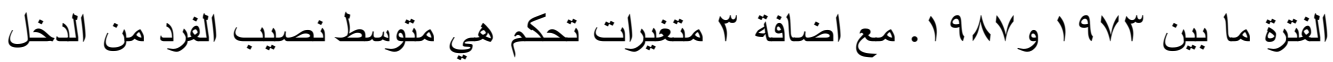

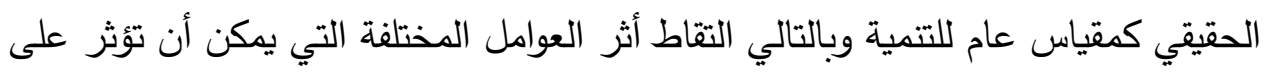

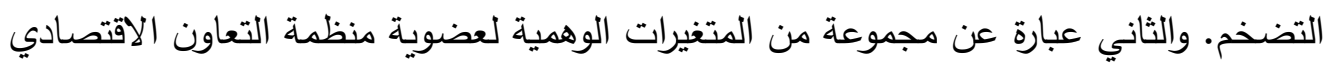


والتتمية وللمناطق المختلفة. ثالثًا متغير وهملاستخدام مؤشر أسعار المستهلكين بدلاً من مكمش الناتج المحلي الإجمالي لقياس التضخم.

وفسر هذه العلاقة العكسية التي توصل اليها بأن الانفتاح التجاري يضع قيودًا على حافز

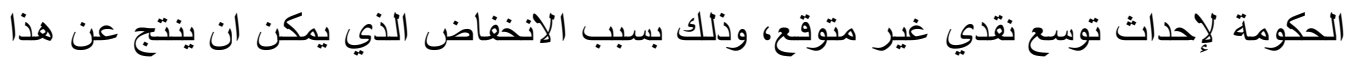
التوسع النقدي في سـعر الصـرف وهو مـا قد يترتب عليـة ارتفاع كبير في مستوي الاسـعار المحلية، وبالتالي من المتوقع أن تحجم الحكومـة عن القيام يمثل هذا التوسع النقدي في ظل سل وجـود الانفتـاح التجـاري. اي ان الانفتـاح التجـاري يضـع قيـودا علـي الحكومـات في استخدام

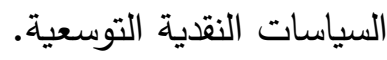

وقد أظهر ( Romer أن متوسط معدل التضخم يكون أقل بالنسبة للاقتصـادات الأصغر والأكثر انفتاحًا نسبيًا. كما أن العلاقة بين التضخم والانفتاح التجاري تكون أقوى في لتصني الدول الأقل استقرارًا من الناحية السياسية وذات البنوك المركزية الأقل استقلالية.

و قد خضعت هذه الفرضية للفحص و الاختبار للعديد من معاصري Romer حاولوا في ذلك التحقق من صحتها او وضع شروط اكثر صرامة لحدود تحققها زمنيا أو مكانيا. وكان من أبرز

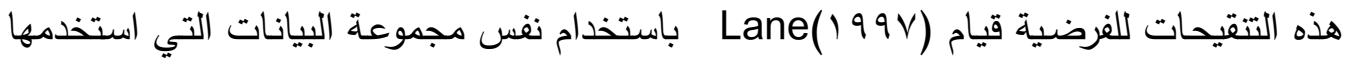

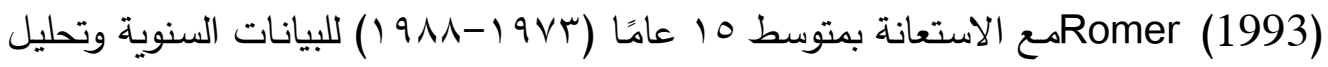
بيانات هذا المقطع العرضي للمتوسطات، توصل أيضًا للعلاقة العكسية بين التضخم والانفتاح. مع التأكيد على أن تأثير الانفتاح كان أقوى عندما تم تضمين حجم الدولة كمتغير تحكم. و من ناحية اخرى توصلت Terra (1998) الى أن العلاقة العكسية بين التضخم والانفتاح

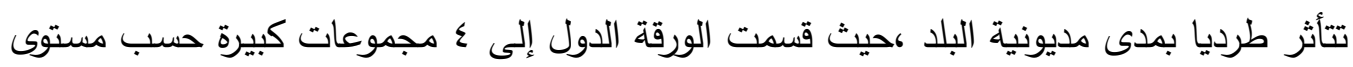




إلى نفس الفائض التجاري لإجراء مدفوعات خدمة الدين الخـارجي. بافتراض تطابق المرونـة

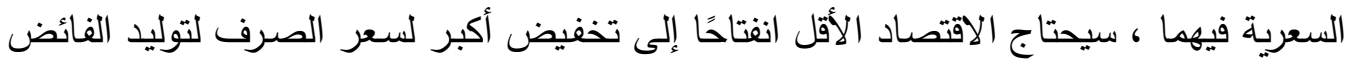
التجاري. ويؤدي خفض قيمة العملة ، بدوره ، إلى زيادة قيمة الالتزامات الخارجية مقومة بالعملة

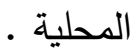

و يجادل المعارضون لهذه الفرضية بأن الانفتاح التجاري لا يقلل بالضرورة من التضخم ، فمن

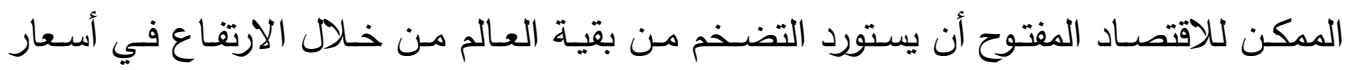

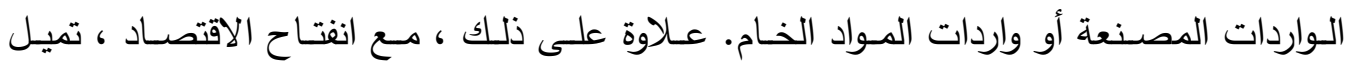
السلطات المالية والنقديـة إلى فقدان قدرتها على السيطرة على التضخم مـن خـلال السياسـات المالية والنقدية (Zakaria, 2010).

كما يلاحظ ان انخفاض قيمة العملة المحلية استجابة لأي صدمة خارجية يمكن أن يؤثر

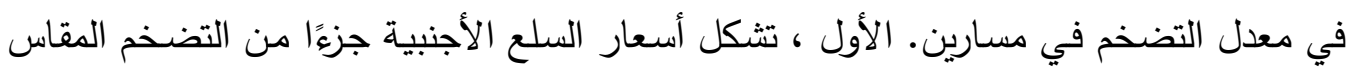

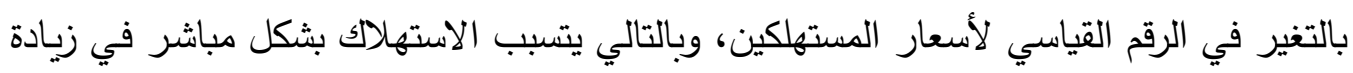
هذا التضخم. و المسار الثاني يتمثل في الزيادة الناشئة عنه في تكاليف انتاج الثركات المحلية.

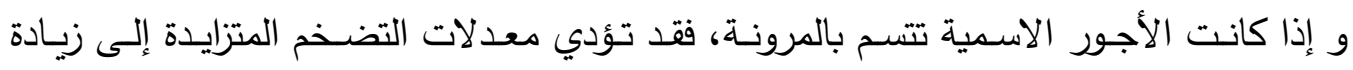

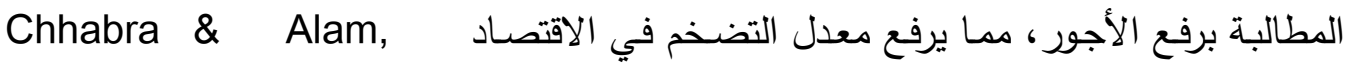

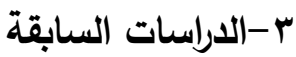

بالرغم من ان فرضية Romer تم بناؤها و انتقادها من قبل معاصريها باستخدام تحليل

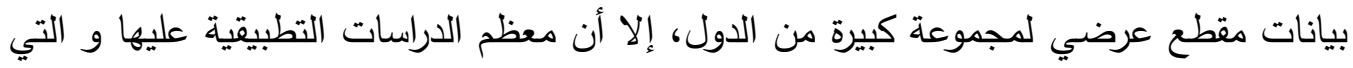
تمت فيما بعد قد اعتمدت على بينات سلاسل زمنية Time Series Data و بيانات ما يطلق 
عليه سلاسل مقطعية (بيانات مقطع عرضي متكرر عبر الزمن) Panel Data . و بالتالي تم تقسيم عرض الدراسات السابقة الى هذين النوعين كما يلي:

ب- ب إسات استخدمت سلاسل مقطعية Panel Data

تتوعت الفترات الزمنية ومجموعات الدول التي شملتها الدراسات في هذا الدجال، كمـا

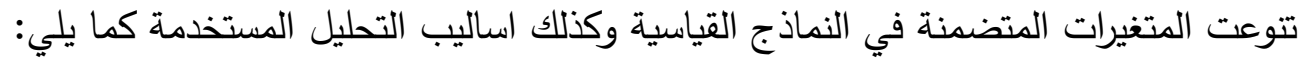
وجد (Ashra أنه إلى جانب المتغيرات الكلية المعتادة مثل معدل نمو النقود ونمو الناتج الزراعي ، كان لمتغيرات الانفتاح (مقاسة بنسبة الصـادرات إلى الناتج المحلي الإجمالي ونسبة الواردات إلى الناتج المحلي الإجمالي) تأثير كبير على التضخم التعاج المحلي في بيانات سلسلة

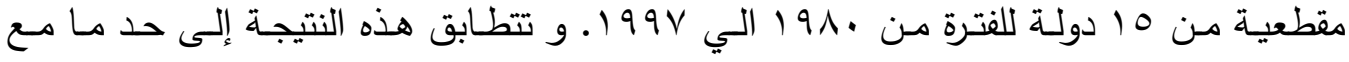

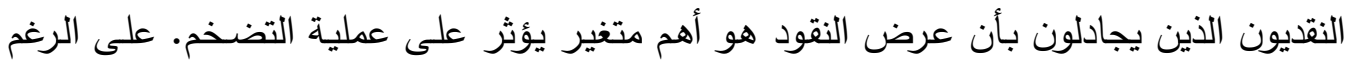

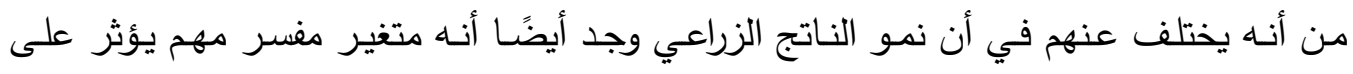

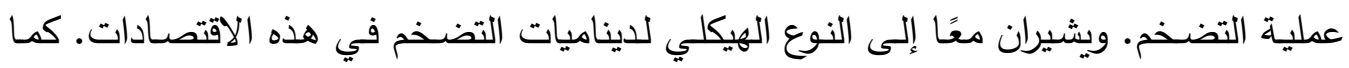
لاحظ أن متغيرات الانفتاح تؤثر بشكل كبير على معدل التضخم ولكن بطريقة معاكسة لبعضها البعض. ففي حين تعمل زيادة نسبة الواردات الى الناتج على تقليل الضغط التضخمي، تميل

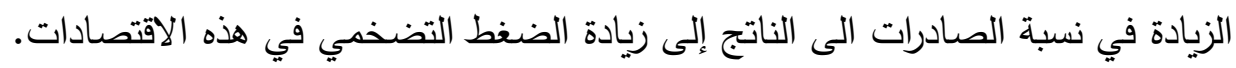

أما Kim and Beladi (2005 فقد قدر العلاقة بين الانفتاح التجاري ومستوى الأسعار

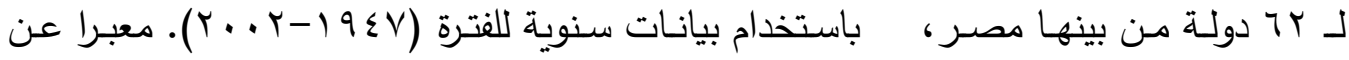

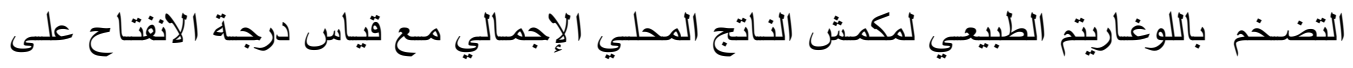
أساس معدل الواردات إلى الناتج المحلي الإجمالي، مع اضافة متغيرات تحكم تتمثل في لوغاريتم

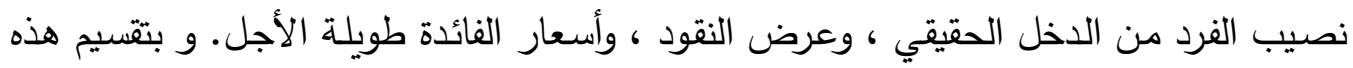

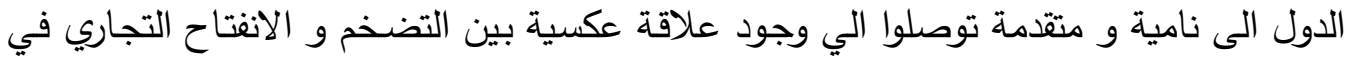


الدول النامية و علاقة طردية بينهما في الدول المتقدمة و كذلك الي ان درجة استقلالية البنوك المركزية لا تلعب دورا جوهريا في تنسير هذه العلاقة.

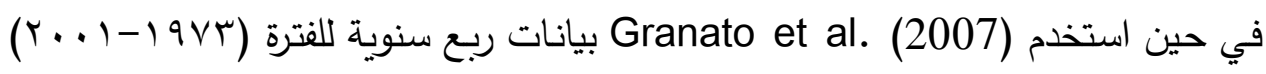

لعدد 9 ادولة من الدول المتقدمة. معبرا عن التضخم باستخدام مؤشر الرقم القياسي لأسعار

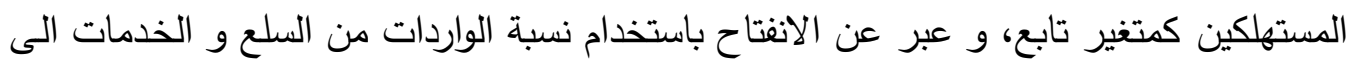

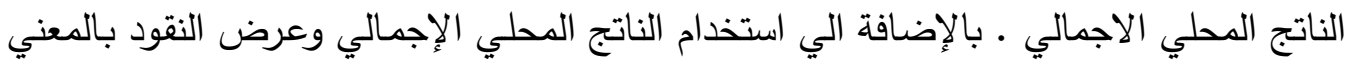

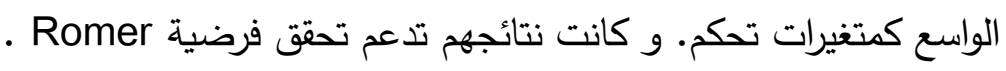

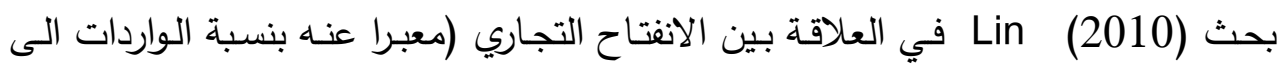

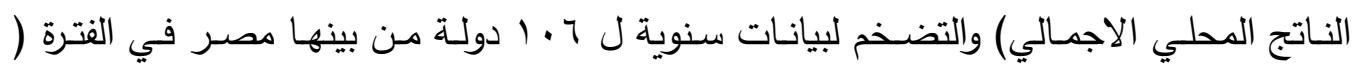

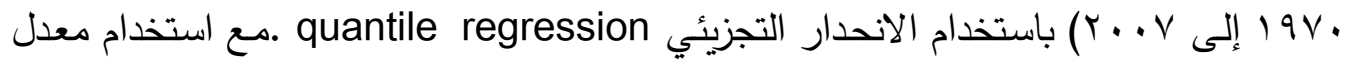

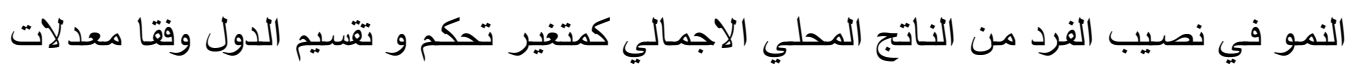

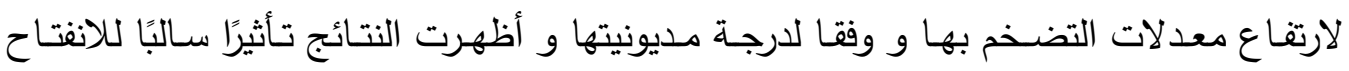
التجاري على التضخم عندما يكون التضخم مرتفع وكنلك في البلدان المدينة في أزمة الديون في

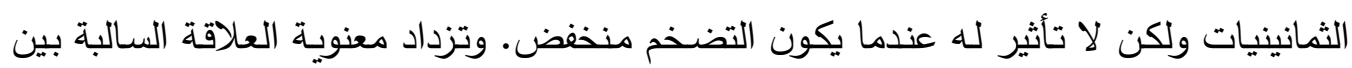
الانفتاح والتضخم بإخال سعر الصرف كمتغير تحكم. لهاير.

واستخدم (Thomas, 2012) بيانات مجمعة لثمانية دول من منطقة الكاريبي خـلال

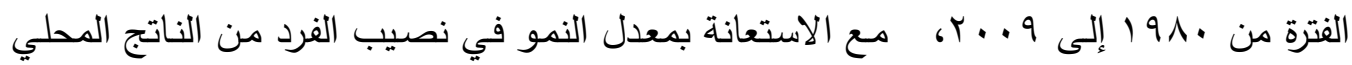



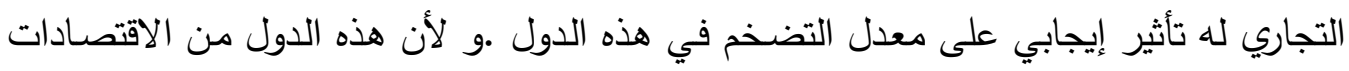

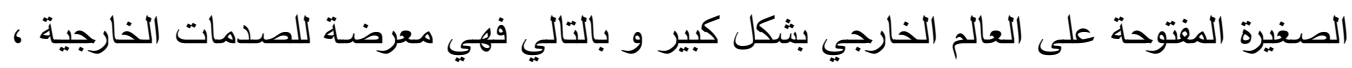
فقد كان ذلك تبريره لرفض فرضية (ب99 () ) Romer. 


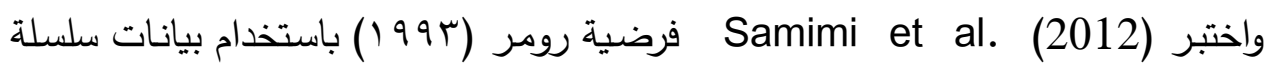

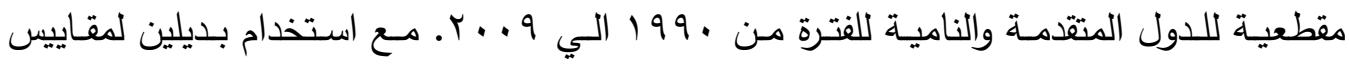
الانفتاح التجاري: المقياس التقليدي للانفتاح التجاري ومقياس أوسع للعولمة الاقتصادية (مؤشر KOF

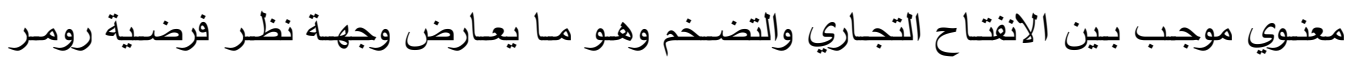

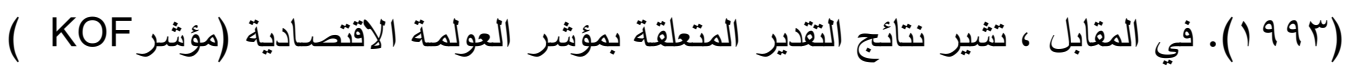

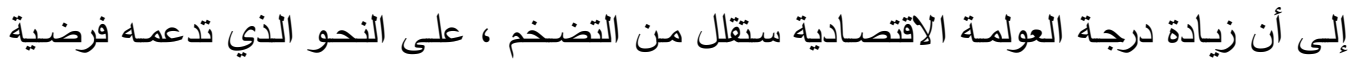

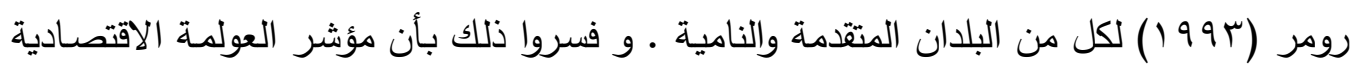

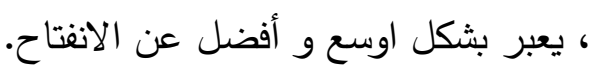

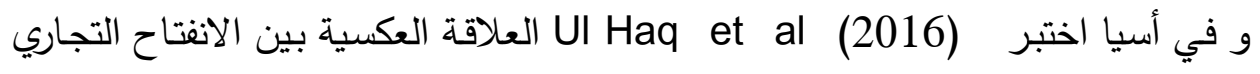
والتضخم، باستخدام عينة من الدول عددها VI دولة من دول آسيا تم تقسيمها إلى مجموعتين

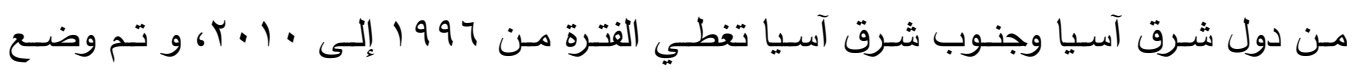

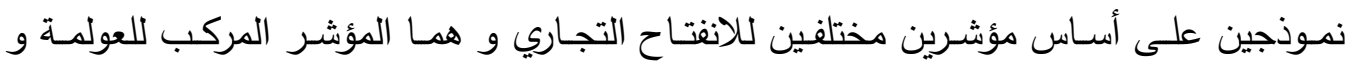
المؤشر التقليدي للانفتاح التجاري و ذلك لاختبار حساسية النتائج للتغيير في مؤشرات الانفتاح

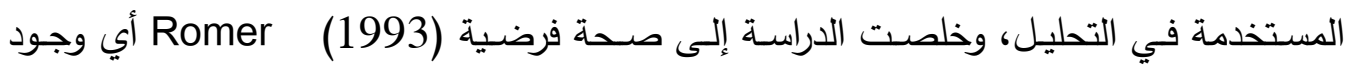
العلاقة العكسية بين الانفتاح التجاري والتضخم في اتجاه واحد فقط باستخدام كلا المؤشرين.

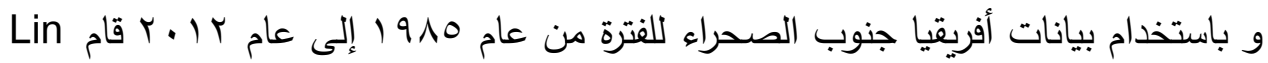

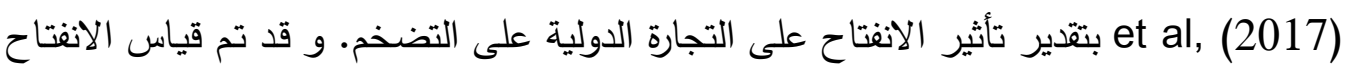

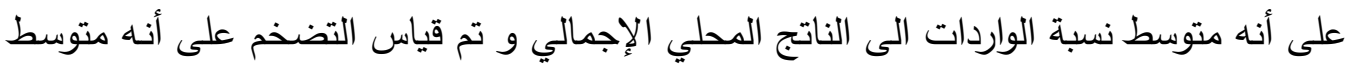

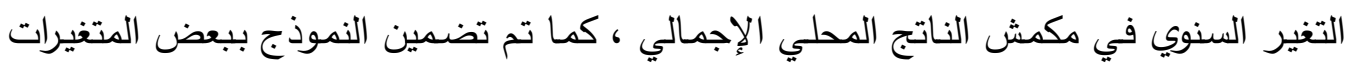

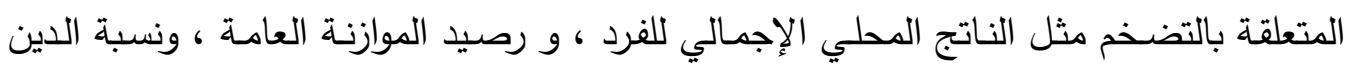

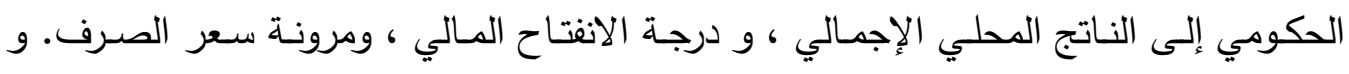


أشارت نتائجهم إلى تحقق الفرضية، حيث ترتبط زيادة نقطة مئوية واحدة في نسبة التجارة الى

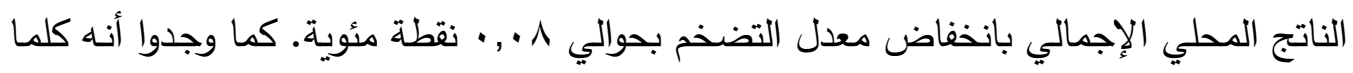

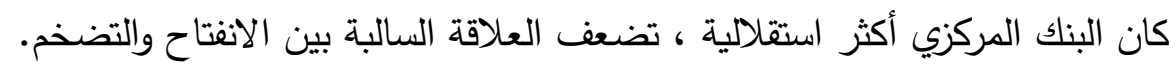
و اخيرا، استخدم Nasrat بيانات سلسلة مقطعية لستة من دول جنوب أسيا

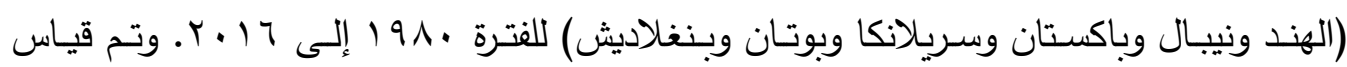

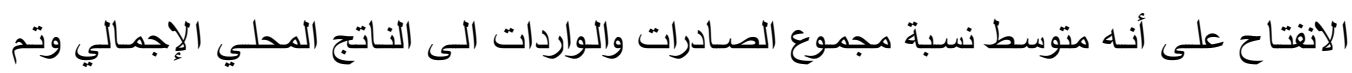
قياس التضخم على أنه متوسط التغير السنوي في الرقم القياسي لأسعار المستهلكين. مستعينا

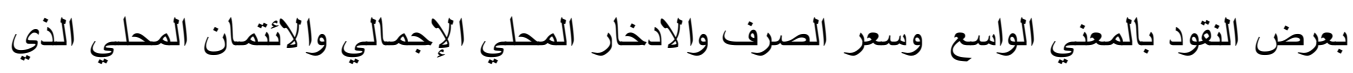

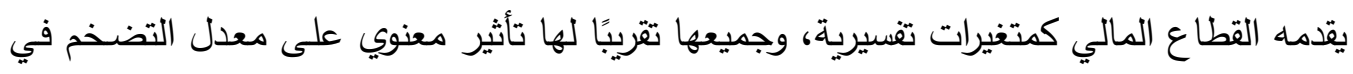

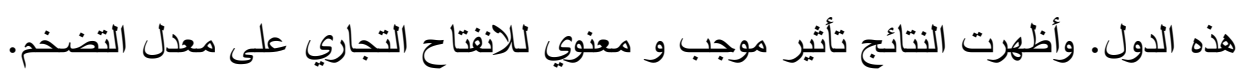

r-r ب دراسات استخدمت سلاسل زمنية لدولة وحيدة

حيث ان الدراسة الحالية تستخدم تحليل السلاسل الزمنية للاقتصساد الدصري ،و حيث ان قد استتنج ان الفرضية تحقق بشكل اخص او أو ضح في اقتصاد صغير فقد تم الاهتمام بشكل التحل

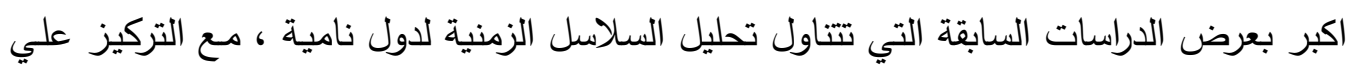
عرض اكثر من دراسة للدولة الواحدة لمحاولة استجلاء مدي الاتفاق او الاختلاف في النتائج باختلاف فترة الدراسة او أسلوب التحليل.

في الهند قام (2010) Joshi \& Acharya بدراسة العلاقة بين الانفتاح التجاري كنسبة

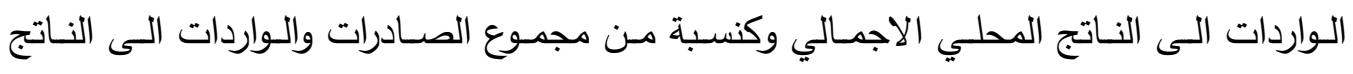

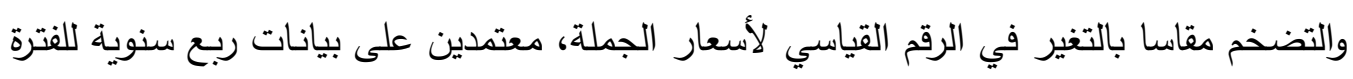

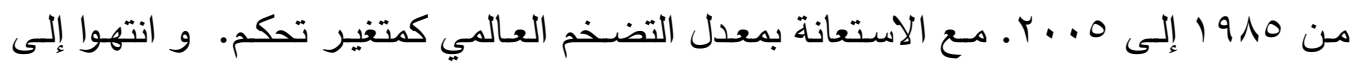

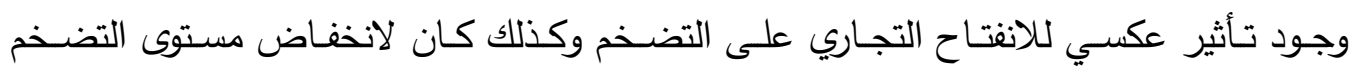




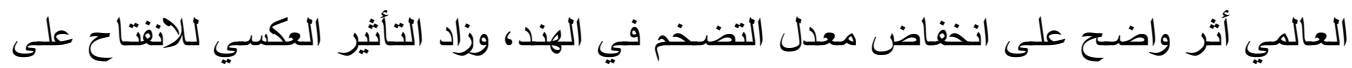
التضخم قوة مع الإصلاح الهيكلي الذي بدأ عام 1919 والتحرير التجاري الكامل.

في حين استخذم Sahu \& Sharma (2018) بيانات ربع سنوية من عام ... T إلى عام 17 ب بتطبيق منهج اختبار الانحدار الذاتي لفترات الابطاء الموزعة (ARDL) ـ وكانت

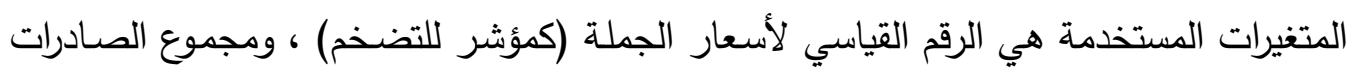
والواردات من السلح والخدمات كنسبة مئويـة مـن النـاتج المحلي الإجمـالي (كمؤشر للانفتـاح



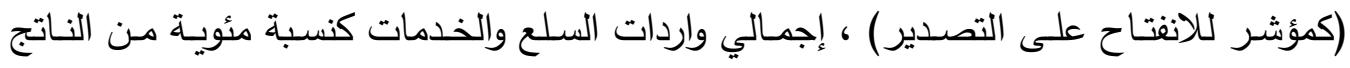

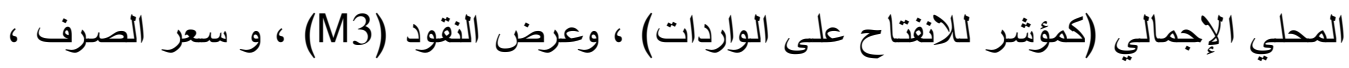

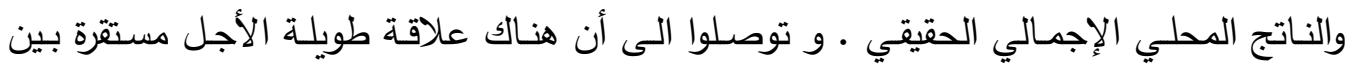

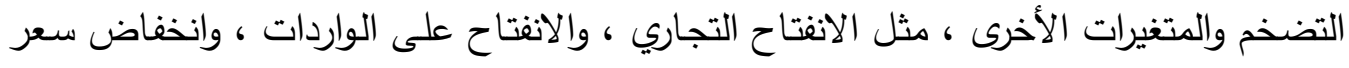

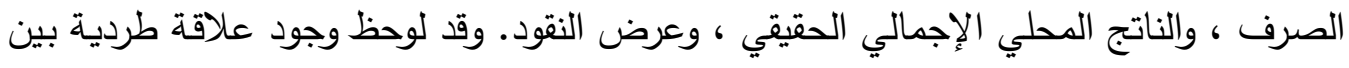

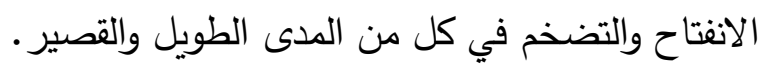

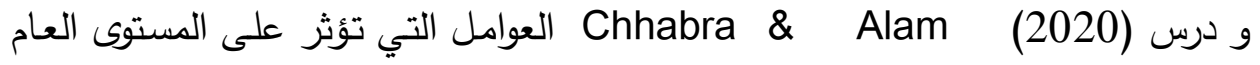

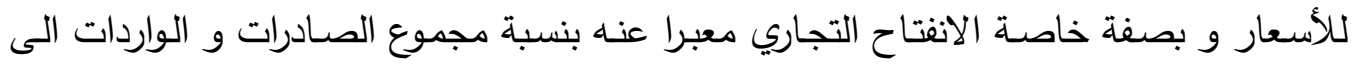

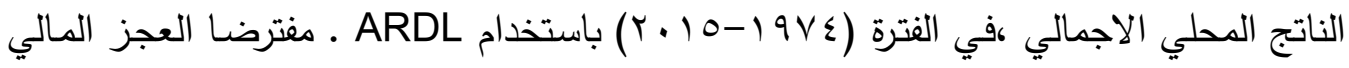
وسـعر الصـرف ومعدل التبادل الدولي كمتغيرات مفسرة للتغير في المستوي العـام للأسعار

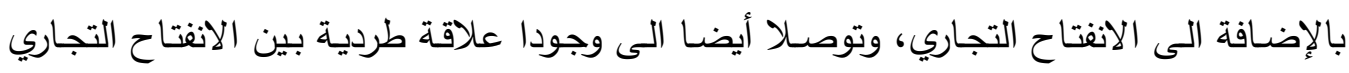
والتضخم اي عدم تحقق الفرضية في الهند.

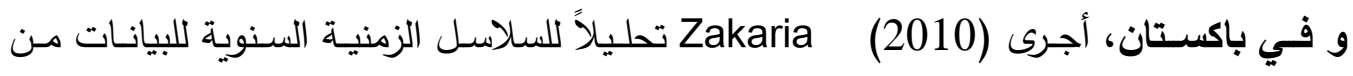

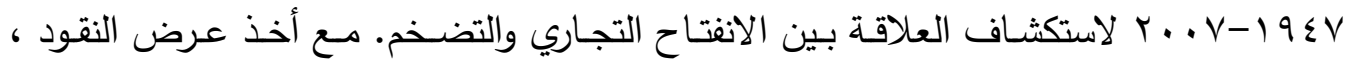

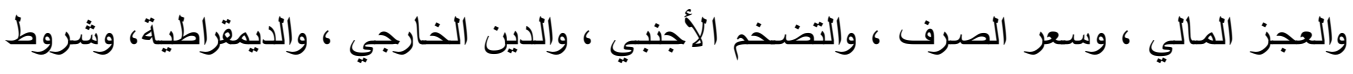


التجارة كمتغيرات تحكم، و توصل الى أن الارتباط طردي بين الانفتاح التجاري والتضخم في

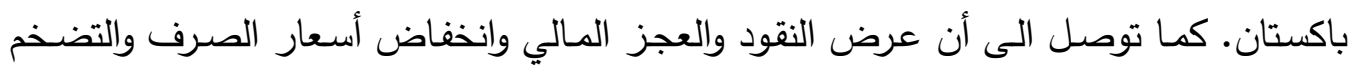

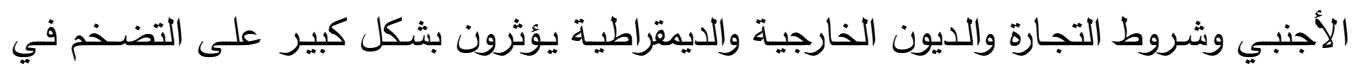
الاتجاهات المتوقعة.

أما Mukhtar (2010) فقد استخدم مدخل التكامل المشترك متعدد المتغيرات ونموذج

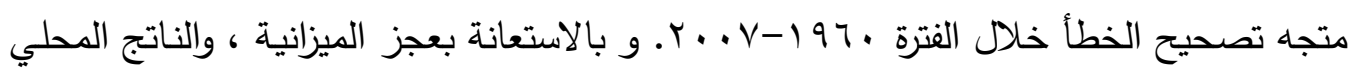

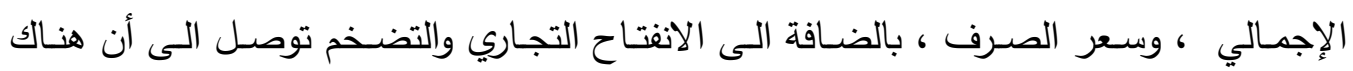

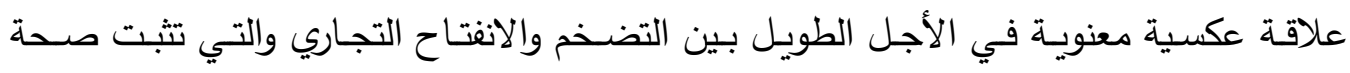
فرضية رومر لباكستان.

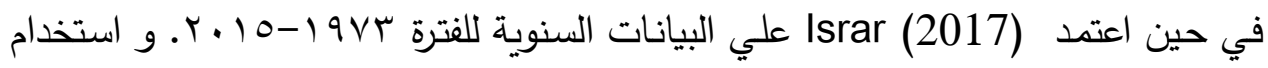

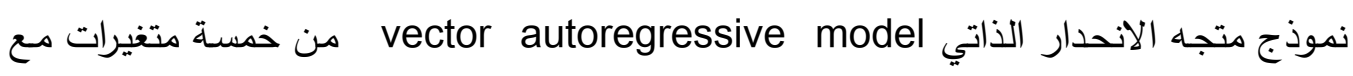
تبديل مختلف للمتغيرات. و توصل الى أنه في الأجل الطويل ، يرتبط الانفتاح ارتباطًا إيجابيًا

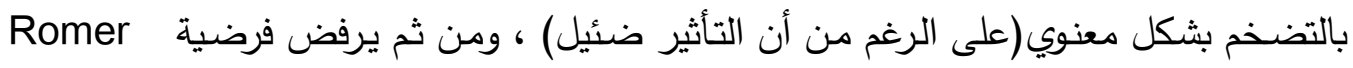

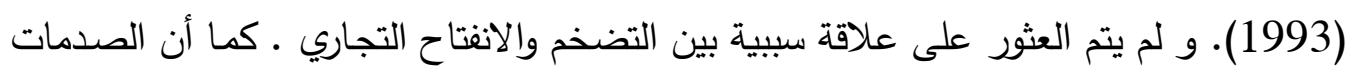

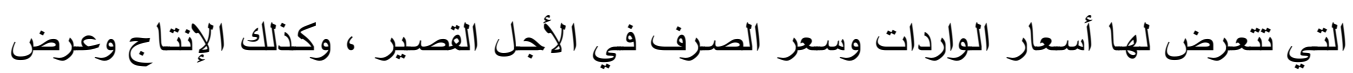
النقود في الأجل الطويل ، لها تأثير على التضخم أكبر من تأثير صدمة الانفتاح.

و يلاحظ أن واردات باكستان تتكون إلى حد كبير مـن المواد الغذائيـة والآلات والسـلع المتعلقة بالطاقة والتي لاحظت ارتفاع الأسعار على مدى عدة سنوات. وقد أدى أدى ذلك إلى مشكلة

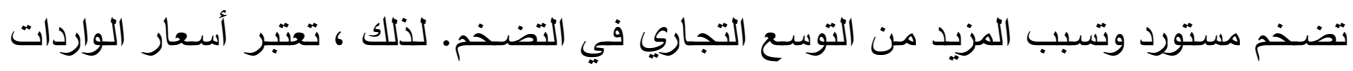

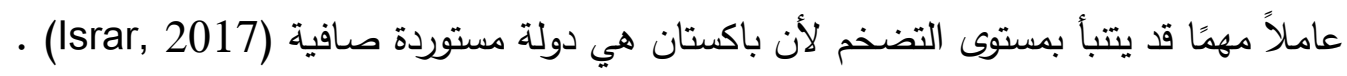
وفي كينيـا استهدف (2016) Wahu دراسة العلاقة بين الانفتاح مقاسـا بصـافي الصـادرات كنسبة مئوية من الناتج المحلي الإجمالي والتضخم مقاسا بالرقم القياسي لأسعار المستهلكين في 


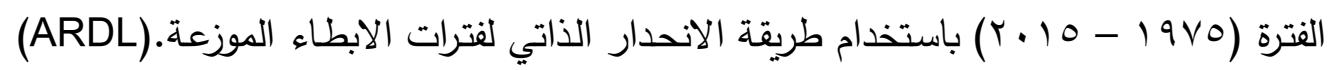

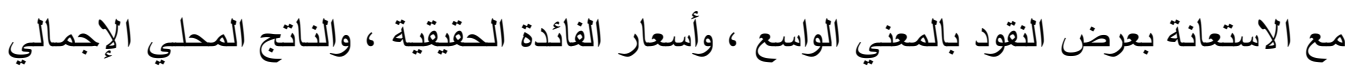

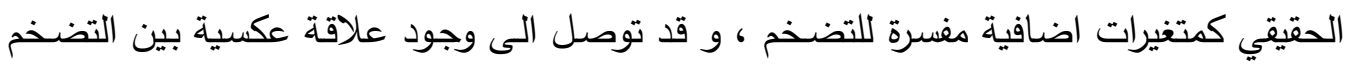
والانفتاح في كينيا وهو ما يدعم تحقق فرضية (1993) Romer في الاقتصاد الكيني و لكن

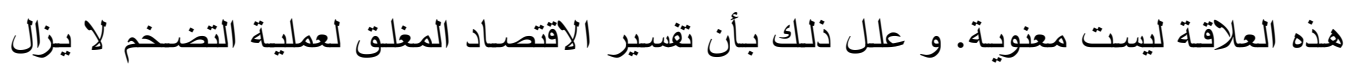
ساريًا.

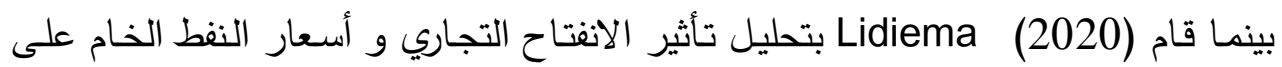

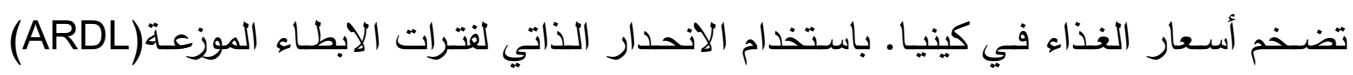

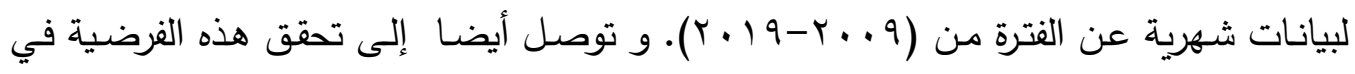



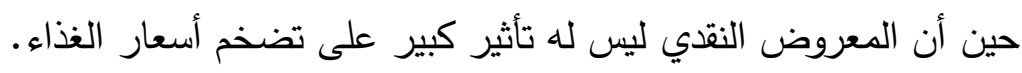

وفي بنجلاديش، قام (2013) باستكثاف العلاقة بين الانفتاح التجاري

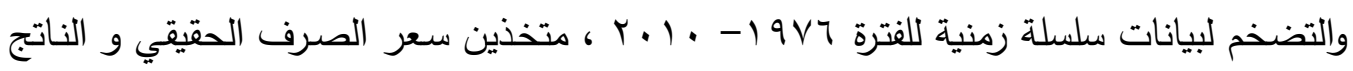

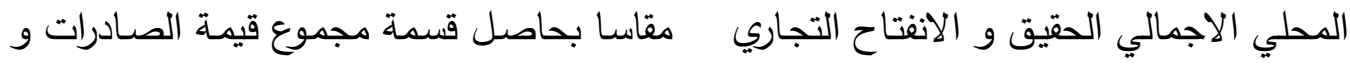

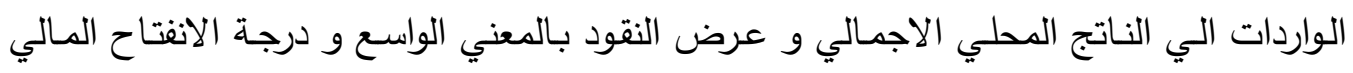

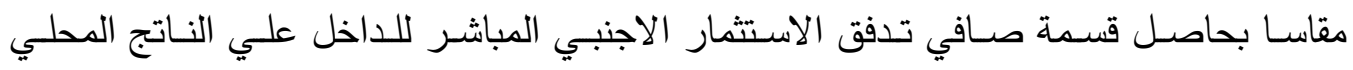



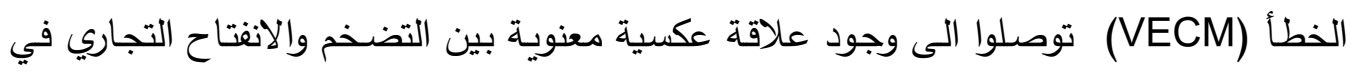
الاجل الطويل فقط.

وفي سريلانكا اختبر (Haq et al (2014) فرضية Romers (1993) مستخدما بيانات

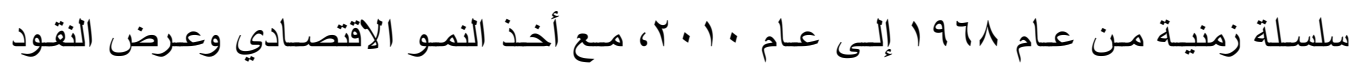

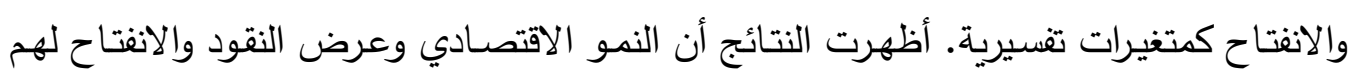


تأثير إيجابي على التضخم وبالتالي ترفض تحقق الفرضية في سريلانكا. و خلصوا إلى أن أن إنيات الانفتاح التجاري قد يؤدي إلى التضخم ، ولكن له تأثير إيجابي على النمو الآقتصادي.

و في تونس بحث Jedidia et al (2019) في العلاقة بين الانفتاح التجاري والتضخم

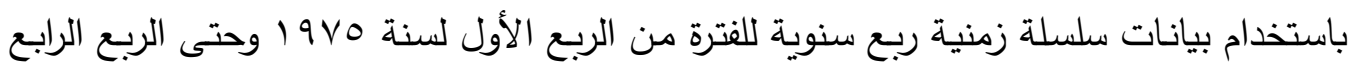

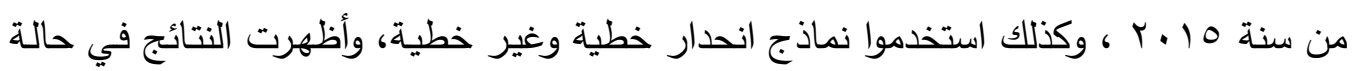

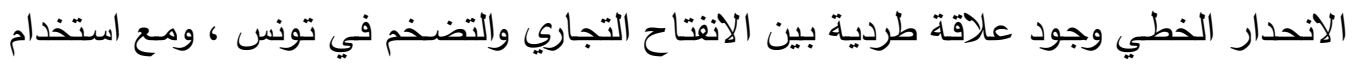

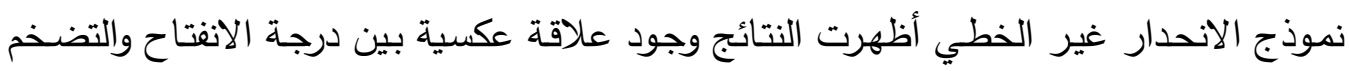

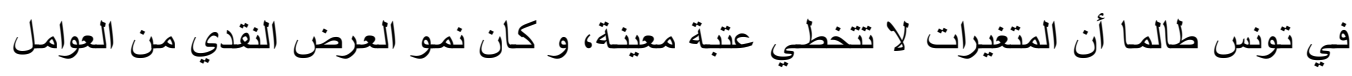

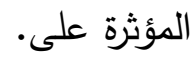

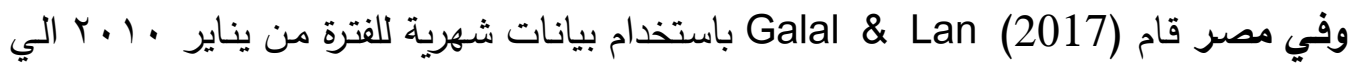

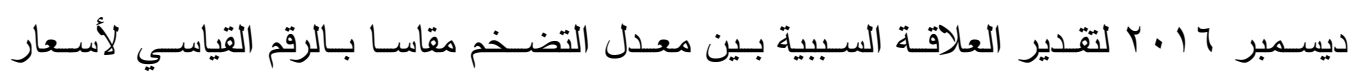
المستهكين والانفتاح التجاري معبرا عنه بمجموع الصادرات والواردات واكتثفا وجود علاقة سبيبة لئية



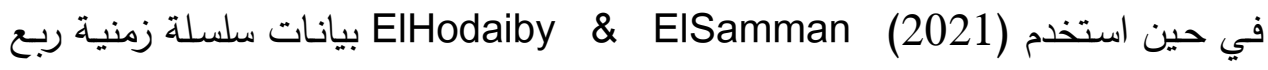

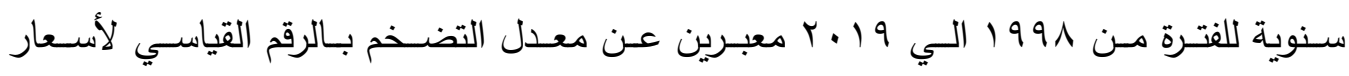

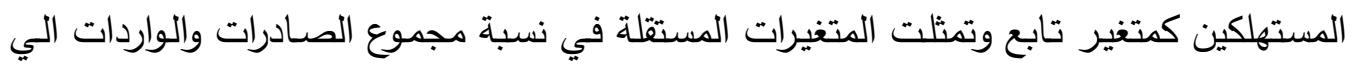

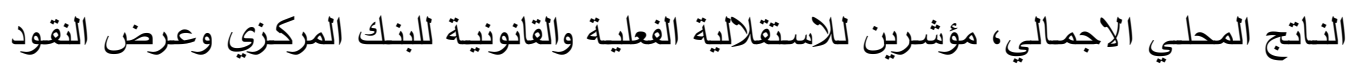

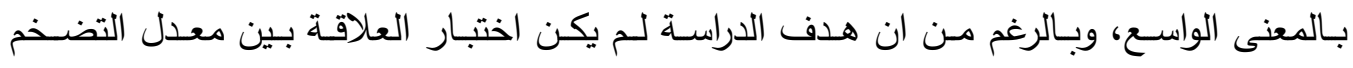
والانفتاح التجاري الا انهما توصلا الي وجود علاقة طردية معنوية إحصائيا بين المتغيرين. 3-3-3: التعليق على الدراسات السابقة 
و من الملاحظ من استعراض الدراسات السابقة أن هناك تعارضا كبيرا بين النتائج التي تم التوصل إليها حتي داخل الدولة الوحدة. ففي الهند على سبيل المثال ، بينما وجد ( Zakaria 2010) و Israr (2017) العلاقة طرديه بين الانفتاح التجاري و التضخم و بالتالي رفضا تحقق الفرضية في الاقتصاد الباكستاني، توصل (2010) Mukhtar الي ان العلاقة عكسية. و و Sahu \& و Chhabra \& Alam لك الحال بالنسبة لباكستان، بينما وجد (2020)

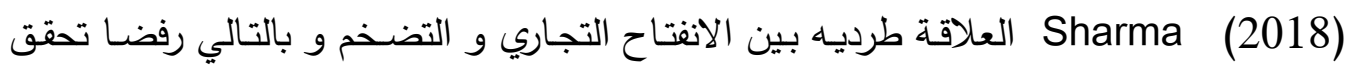
الفرضية في الاقتصاد الباكستاني، توصل (2010) Joshi \& Acharya عكسية. و قد يرجع ذلك الي اختلاف متغيرات التحكم في النموذج او طرق قياس المتغيرات او الي اختلاف الاسلوب المستخدم في تقدير النموذج أو الفترة الزمنية محل الدراسة.

و يعني ذلك ان القضية غير محسومة و ما زال الباب مفتوحا لتحسين النماذج المستخدمة و اساليب التقدير للوصول الى نتائج اكثر دقة. و كذلك يعني خصوصية ظروف كل دولة ولة و اختلاف استجابتها للتغير في درجة الانفتاح الاقتصـادي و هو ما يفتح الباب الى المزيد من البحث.

تعددت متغيرات التحكم الداخلـة في النمـاذج التي استخدمتها الدراسـات السابقة و لكنها دارت حول استخدام متغير يعكس الناتج المحلي الاجمالي الحقيقي او النمو فيه ليوضـح الاثر العكسي المتوقع للزيادة في الانتاج على التضخم. و ايضـا متغير يعكس اثر السياسـة المالية

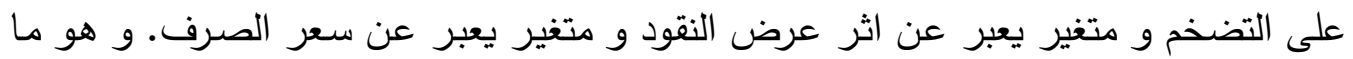
قامت الدراسة الحالية بالسير على نهجه.

انقسمت الدراسات السابقة في قياس درجة الانفتاح الاقتصـادي بين الاعتماد علي نسبة مجموع الصادرات و الواردات الى الناتج المحلي الاجمالي ام استخدام نسبة الواردات الى الناتج 
فقط، في حين استخدم البعض مؤشر العولمة الاقتصادية ليعكس مفهوم اوسع للانفتاح التجاري. و لذلك قامت الدراسة الحالية بتجربة استخدام كل من هذه المقاييس الثلاثة في نموذج على حده

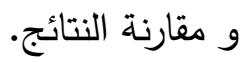

؛ : الاراسة التطبيقية علي مصر

ع - 1: النموذج التطبيقي ووصف المتغيرات.

طبقـال ( Romer يضـع الانفتـاح التجـاري قيودًا على حـافز الحكومـة لإحداث توسع نقدي غير متوقع، وذلك بسبب الانخفاض الذي يمكن ان ينتج عنه في سعر الصرف. و علي ذلك يشتمل النموذج المستخدم في الدراسة الحالية علي المتغيرات التالية:

- معدل التضخم: كان الاعتماد الاساسي في معظم الدراسات التي اختبرت الفرضية على معدل

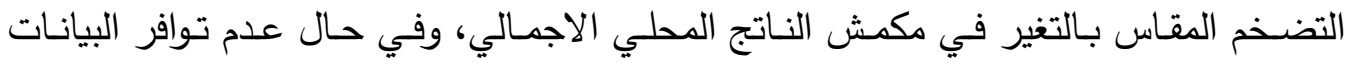

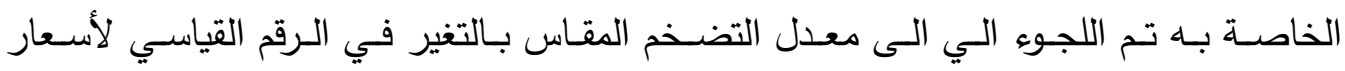

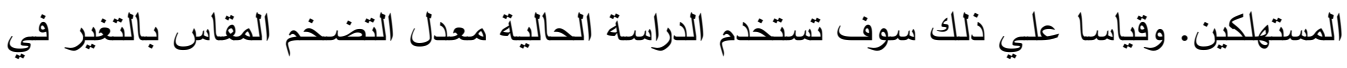

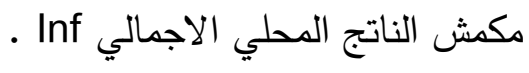

- درجة الانفتاح التجاري: و قد تعددت المؤشرات التي تم استخدامها للتعبير عنه في الدراسات

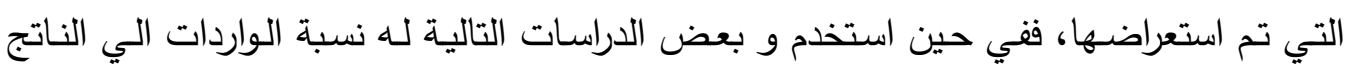

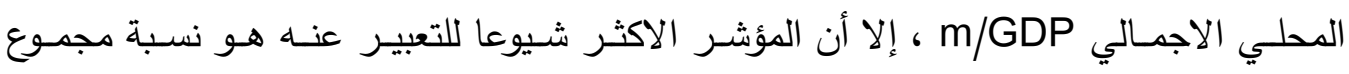

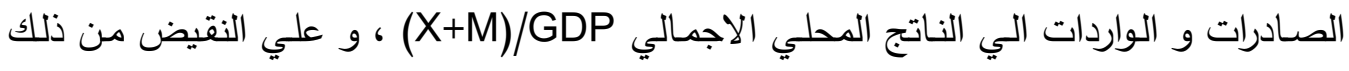

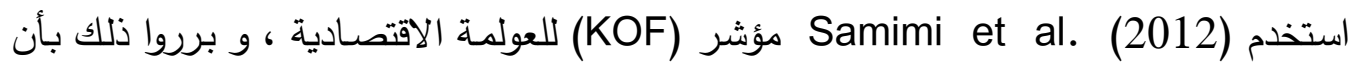
المؤشر التقليدي للانفتاح التجاري و هو (x+ m)/GDP يمثل مؤشر فرعي للعولمة الاقتصادية

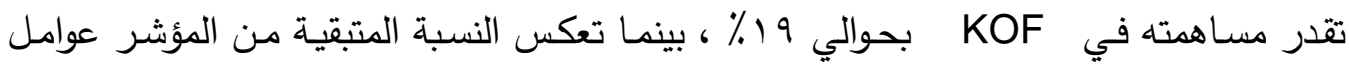

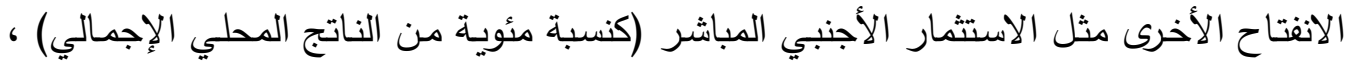


ومدفوعات الدخل للمواطنين الأجانب (كنسبة مئويـة من الناتج المحلي الإجمالي) والقيود التي تمثل حواجز الاستيراد الخفية ، ومتوسط معدل التعريفة ، والضرائب على التجارة الدولية (كنسبة التبائ مئوية من الإيرادات الضريبية) وقيود حساب رأس المال. و على ذلك سوف تقوم الدراسة الحالية باختبار فرضية Romer بالاعتماد على ثلاثة

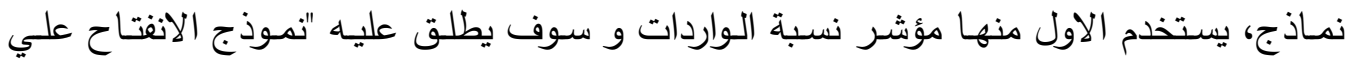

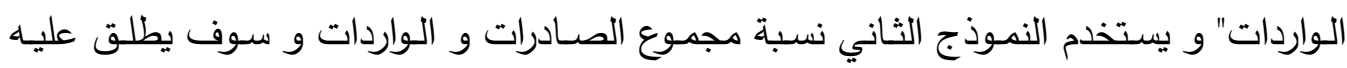

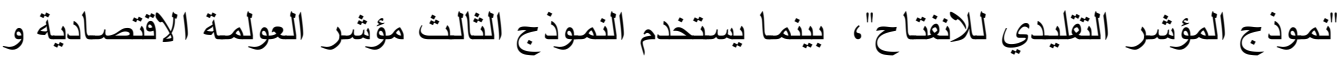

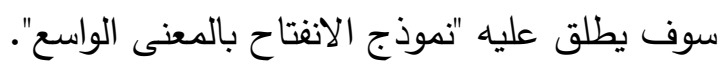

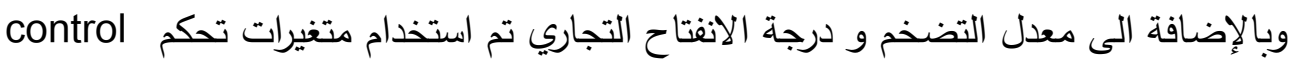
variables الانفتاح التجاري و هذه العوامل هي:

- معدل ندو نصيب الفرد من الناتج المحلي الاجمالي الحقيقي (R_GDP_P_G) و من التصني المتوقع ان يؤثر عكسيا علي التضخم حيث يتضمن النمو الاقتصادي الزيادة في العرض الكلي

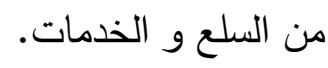

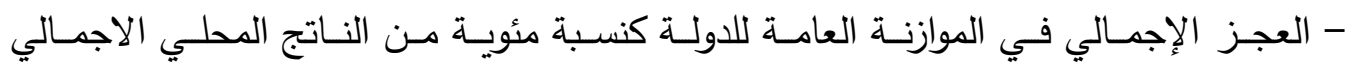

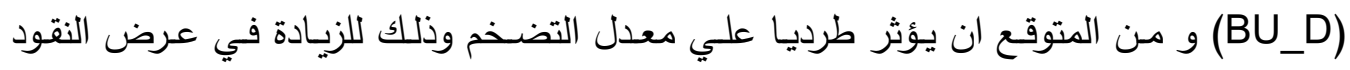

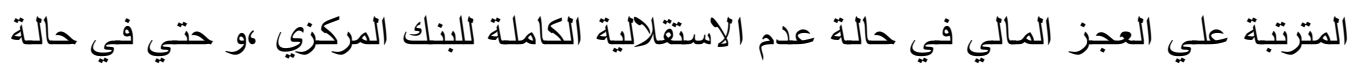

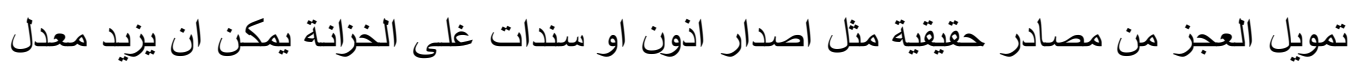

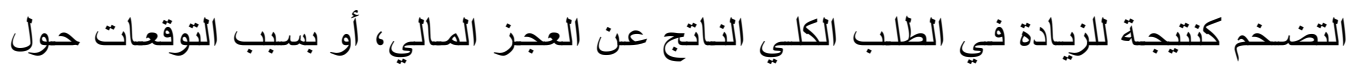
التوسع النقدي في المستقبل(Ashra, 2002) . - متوسط سعر صرف الدولار الامريكي بالجنيهات المصرية (EXC) و هو يعبر عن مقلوب

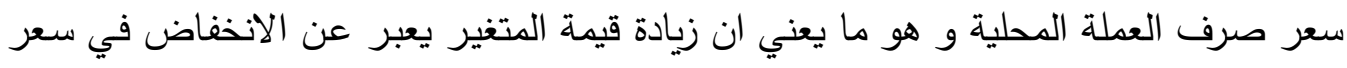


العملة المحلية. و يتوقف تأثيره علي معدل التضخم علي مرونة كل من الصادرات و الواردات للتغير في سعر الصرف، فإذا نتج عن انخفاض قيمة العملة مثلا زيادة في الصادرات فقد يؤدي ذلك الى زيادة الطلب علي السلع المحلية و نقص المعروض منها محليا و بالتالي امكانية ارتفاع

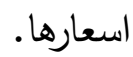

- عرض النقود بالمعني الواسع كنسبة الى الناتج المحلي الاجمالي (M2/GDP) • و يتوقع ان يكون تأثيره طرديا علي معدل التضخم.

يضاف الي متغيرات التحكم هذه نسبة الصادرات الي الناتج المحلي الاجمالي لتعبر عن درجة الانفتاح على الصادرات x/GDP فقط في "نموذج الانفتاح على الواردات" كما في .Lin et al (2017) و (2002) وبالتالي يمكن صياغة النماذج التطبيقية للبحث، كما يلي: أولا: نموذج الانفتاح على الواردات"

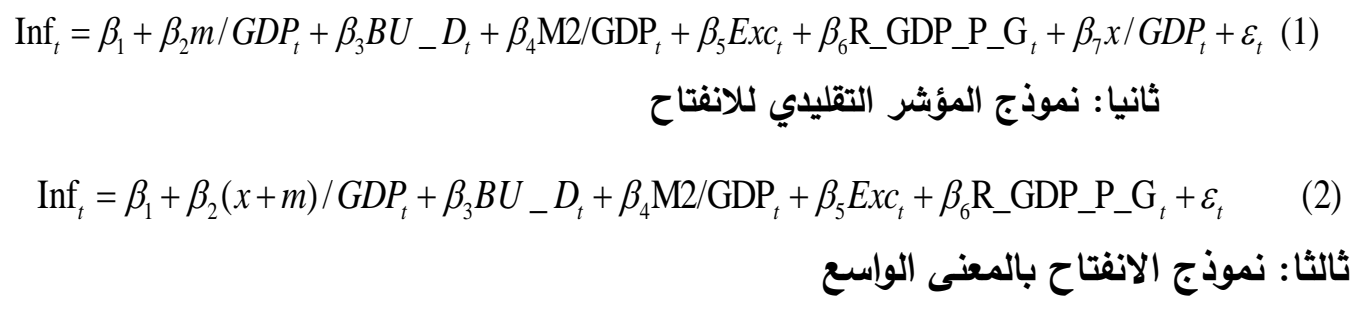
$\operatorname{Inf}_{t}=\beta_{1}+\beta_{2} K O F_{t}+\beta_{3} B U_{-} D_{t}+\beta_{4} \mathrm{M} 2 / \mathrm{GDP}_{t}+\beta_{5} E x c_{t}+\beta_{6} \mathrm{R}_{-} \mathrm{GDP} \mathrm{P}_{-} \mathrm{G}_{t}+\varepsilon_{t}$

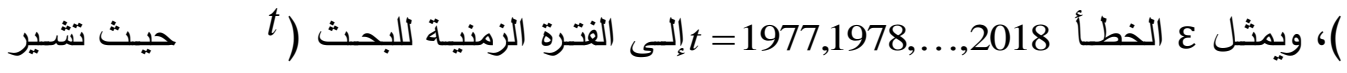
هي معلمات النموذج المراد تقديرها. ومن المتوقع أن تكون

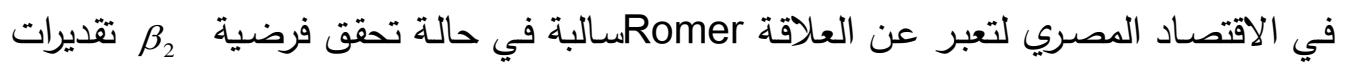

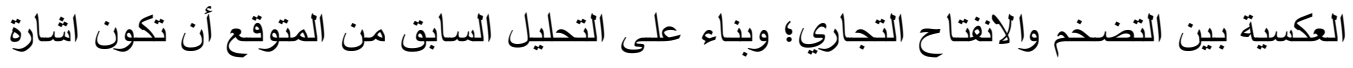

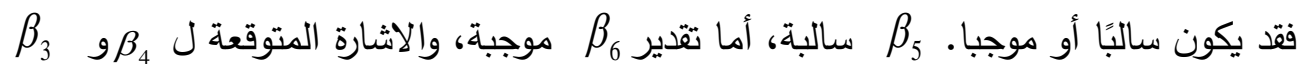


و تختلف الدراسة الحالية عن الدراسات السابقة لهذه العلاقة في الاقتصاد المصري في فترة التقدير و المتغيرات المتضمنة في النموذج و كذلك في الاسلوب المستخدم لتقدير النموذج. بالإضافة إلى التعبير عن متغير الانفتاح التجاري بأكثر من طريقة ومقارنة النتائج.

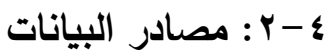

تم الحصـول على جميع البيانـات مـن قاعدة البيانـات الخاصـة بمؤشرات التتميـة الدوليـة World Bank والتي يصدرها البنك الدولي World Development Indicators (WDI) المتاحة على World Development Indicators | Databank (worldbank.org) في حين لم يتم العثور علي سلسلة منتظمة لبيانات العجز في الموازنة العامة للدولة تغطي

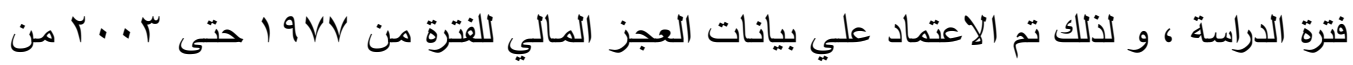
Marinheiro

http://www.mof.gov.eg لإكمال باقي السلسلة الزمنية.

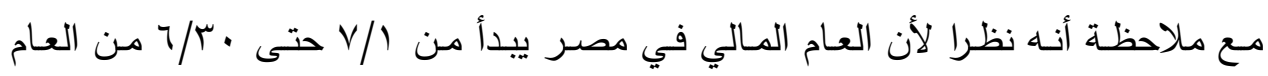

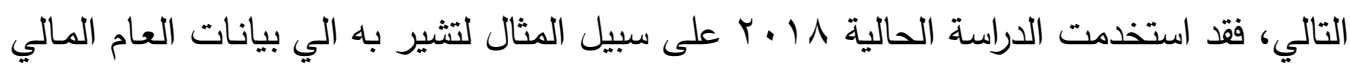
. r. IN/ r. IV

؟ - r: الأسلوب المستخدم لتقدير النموذج

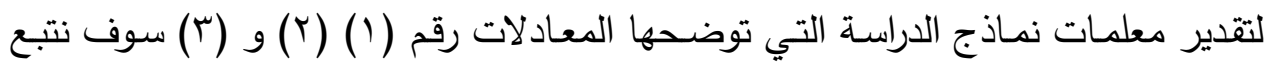

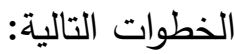

1- نظرا لاعتماد البحث على بيانات سلاسل زمنية، فإن الخطوة الأولي تتمثل في اختبار مدي

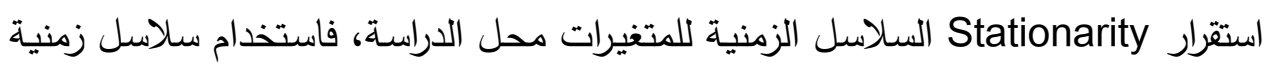
غير مستقرة في الانحدار يترتب عليه مشكلة الانحدار الزائف Spurious Regression، حيث يؤدي استخدام طريقة المربعات الصغرى العادية الى نتائج غير صحيحة، ففي هذه

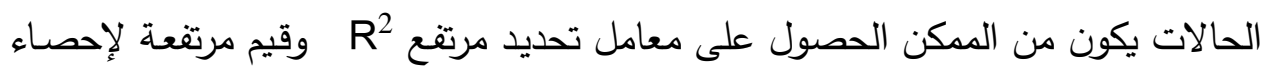


t في حين أن المتغيرات المستخدمة في الانحدار لا تربطها أي علاقة. و قد تم استخدام



الوحدة (Dickey and Fuller 1979) لاختبار استقرار هذه السلاسل الزمنية.

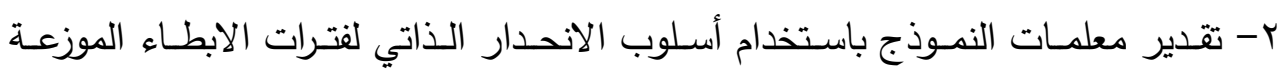
Autoregressive Distributed Lags (ARDL) الششترك Bound Testing for Cointegration . و قد تم استخدام هذا الأسلوب لأنة يمكن استخدامه في حالة اختلاف رتب التكامل بين المتغيرات بحيث

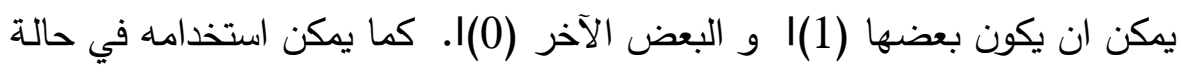

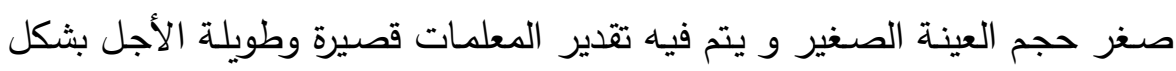

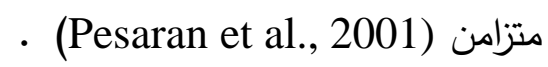

$$
\begin{aligned}
& \text { ؛ - ؛ : نتائج تقدير النماذج } \\
& \text { ع - ع- 1: اختبارات جذر الوحدة }
\end{aligned}
$$

الجدول التالي رقم (1) يوضح نتائج اختبارات جذر الوحدة لجميع المتغيرات الموجودة في

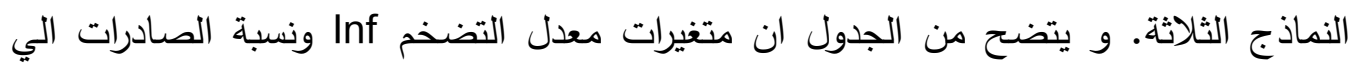
الناتج المحلي الاجمالي x/GDP و عرض النقود بالمعنى الواسع كنسبة الى الناتج المحلي الاجمالي M2/GDP و نسبة مجموع الصادرات و الواردات الي الناتج المحلي الاجمالي (X+M)/GDP العامة BU_D و مؤشر العولمة الاقتصادية KOF و نسبة الواردات الي الناتج المحلي الاجمالي m/GDP و سعر الصرف EXC كانت غير مستقرة في المستوي و لكنها تصبح مستقرة بعد أخذ الفرق الأول، أي أنها متكاملة من الرتبة (1)ا ، و هو ما يدعم ضرورة استخدام أسلوب الانحدار الذاتي لفترات الابطاء الموزعة في التقدير . 


\section{جدول رقم (1) نتائج إختبار جذر الوحدة الموسع لايكي - فولر - Augmented Dickey}

Fuller (ADF)

\begin{tabular}{|c|c|c|c|}
\hline Inf & & t-Statistic & Prob.* \\
\hline \multicolumn{2}{|c|}{ Augmented Dickey-Fuller test statistic } & -4.657879 & 0.0005 \\
\hline \multirow{3}{*}{ Test critical values: } & $1 \%$ level & -3.600987 & \\
\hline & $5 \%$ level & -2.935001 & \\
\hline & $10 \%$ level & -2.605836 & \\
\hline \multicolumn{2}{|l|}{ BU_D } & t-Statistic & Prob.* \\
\hline \multicolumn{2}{|c|}{ Augmented Dickey-Fuller test statistic } & -0.996694 & 0.2809 \\
\hline \multirow[t]{3}{*}{ Test critical values: } & $1 \%$ level & -2.622585 & \\
\hline & $5 \%$ level & -1.949097 & \\
\hline & $10 \%$ level & -1.611824 & \\
\hline \multicolumn{2}{|l|}{ D(BU_D) } & t-Statistic & Prob.* \\
\hline \multicolumn{2}{|c|}{ Augmented Dickey-Fuller test statistic } & -7.570466 & 0.0000 \\
\hline \multirow[t]{3}{*}{ Test critical values: } & $1 \%$ level & -2.624057 & \\
\hline & $5 \%$ level & -1.949319 & \\
\hline & $10 \%$ level & -1.611711 & \\
\hline \multicolumn{2}{|l|}{$\mathrm{X} / \mathrm{GDP}$} & t-Statistic & Prob. ${ }^{*}$ \\
\hline \multicolumn{2}{|c|}{ Augmented Dickey-Fuller test statistic } & -5.159649 & 0.0001 \\
\hline \multirow[t]{3}{*}{ Test critical values: } & $1 \%$ level & -3.621023 & \\
\hline & $5 \%$ level & -2.943427 & \\
\hline & $10 \%$ level & -2.610263 & \\
\hline \multicolumn{2}{|l|}{ KOF } & t-Statistic & Prob.* \\
\hline \multicolumn{2}{|c|}{ Augmented Dickey-Fuller test statistic } & -0.542380 & 0.4759 \\
\hline \multirow[t]{3}{*}{ Test critical values: } & $1 \%$ level & -2.622585 & \\
\hline & $5 \%$ level & -1.949097 & \\
\hline & $10 \%$ level & -1.611824 & \\
\hline \multicolumn{2}{|l|}{$\mathrm{D}(\mathrm{KOF})$} & t-Statistic & Prob.* \\
\hline \multicolumn{2}{|c|}{ Augmented Dickey-Fuller test statistic } & -7.123755 & 0.0000 \\
\hline Test critical values: & $1 \%$ level & -2.624057 & \\
\hline
\end{tabular}




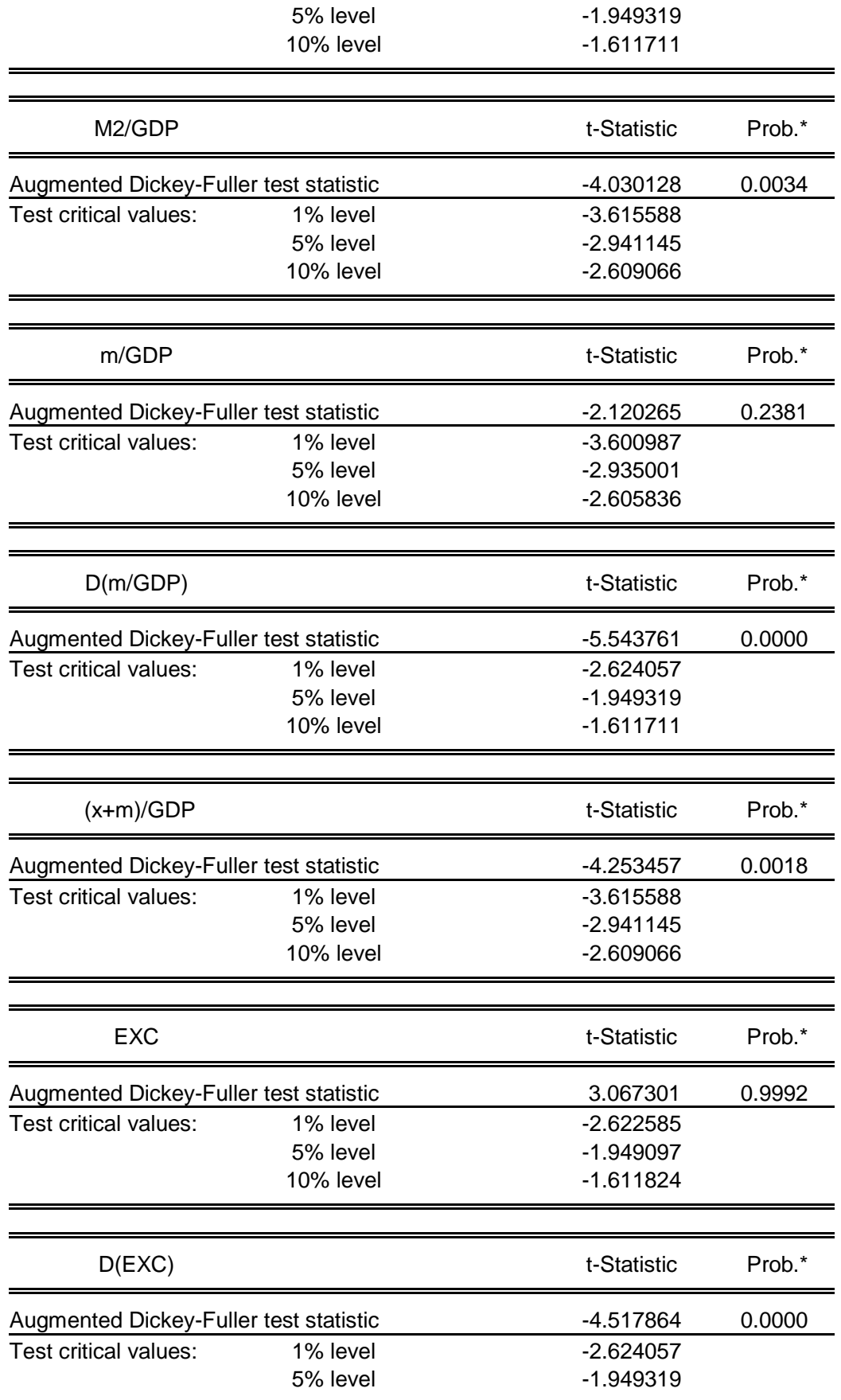




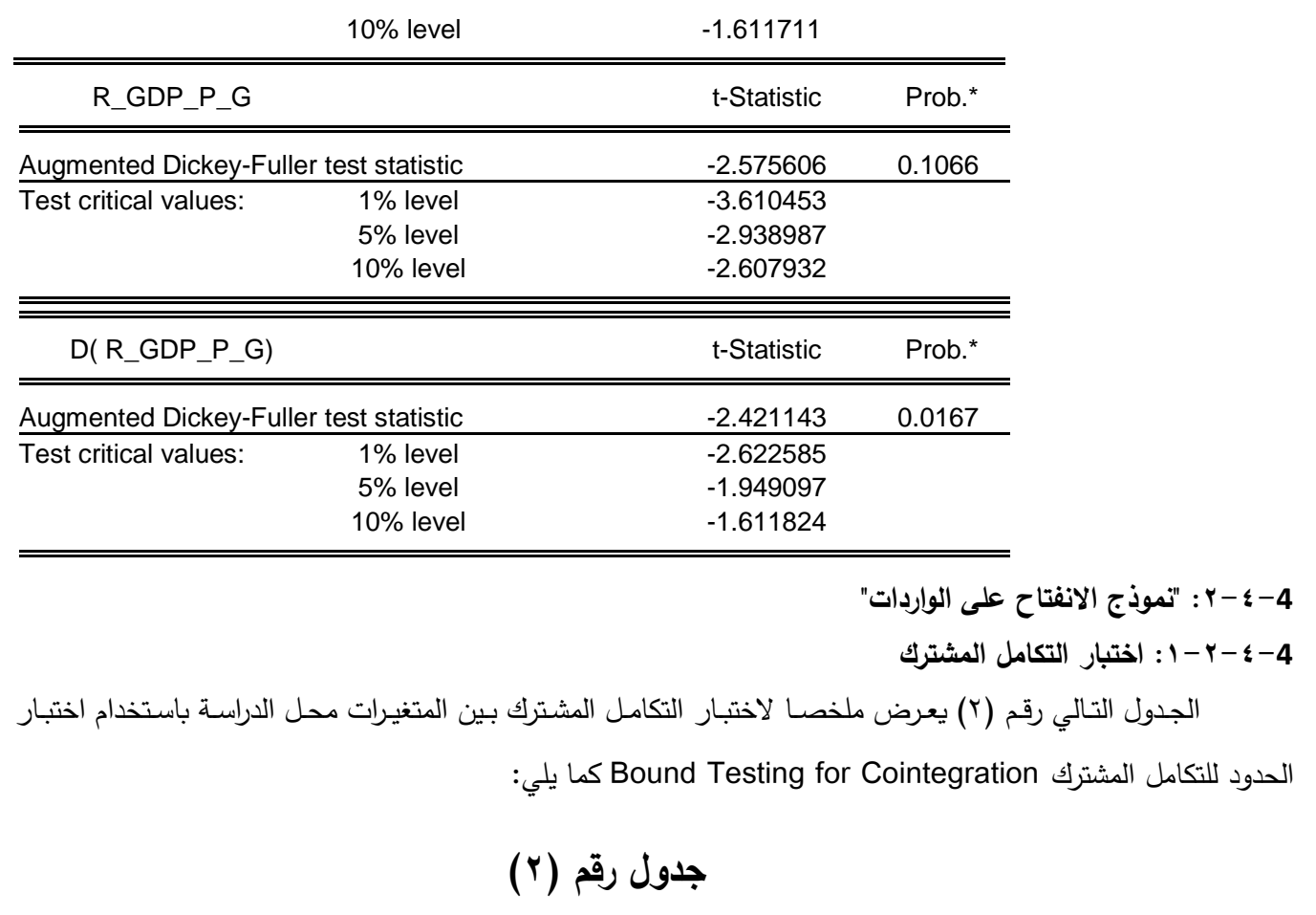

Bound Testing for Cointegration نتائج اختبار التكامل المشترك

\begin{tabular}{lcccc} 
F-Bounds Test & & \multicolumn{2}{c}{$\begin{array}{r}\text { Null Hypothesis: No levels } \\
\text { relationship }\end{array}$} \\
\hline \hline Test Statistic & Value & Signif. & $\mathrm{I}(0)$ & $\mathrm{I}(1)$ \\
\hline \hline F-statistic & 13.68527 & $10 \%$ & 1.75 & 2.87 \\
k & 6 & $5 \%$ & 2.04 & 3.24 \\
& & $2.5 \%$ & 2.32 & 3.59 \\
& & $1 \%$ & 2.66 & 4.05 \\
\hline \hline
\end{tabular}

و يتضح من الجدول أن F- Statistic قد بلغت قيمتها 13.68527 وهى أعلى من القيمة الجدولية الحرجة ، للحد

الأعلى، عند مستوى معنوية ا (\% و بالتالي يُمكن رفض فرض العدم، الذى ينص على عدم وجود علاقة طويلة الأجل بين متغيرات النموذج. أي أنه يوحد علاقة تكامل مُشترك Cointegration طويلة الأجل بين المتغيرات محل الدراسة.

צ - ـ - - - : تقدير معلمـات النموذج وقبل استخدام أسلوب الانحدار الذاتي لفترات الابطاء الموزعـة ARDL لتقدير

Akaike Information معلمات النموذج تم تحديد العدد الأمثل لفترات الابطاء اللازمة لكل متغير باستخدام معيار 


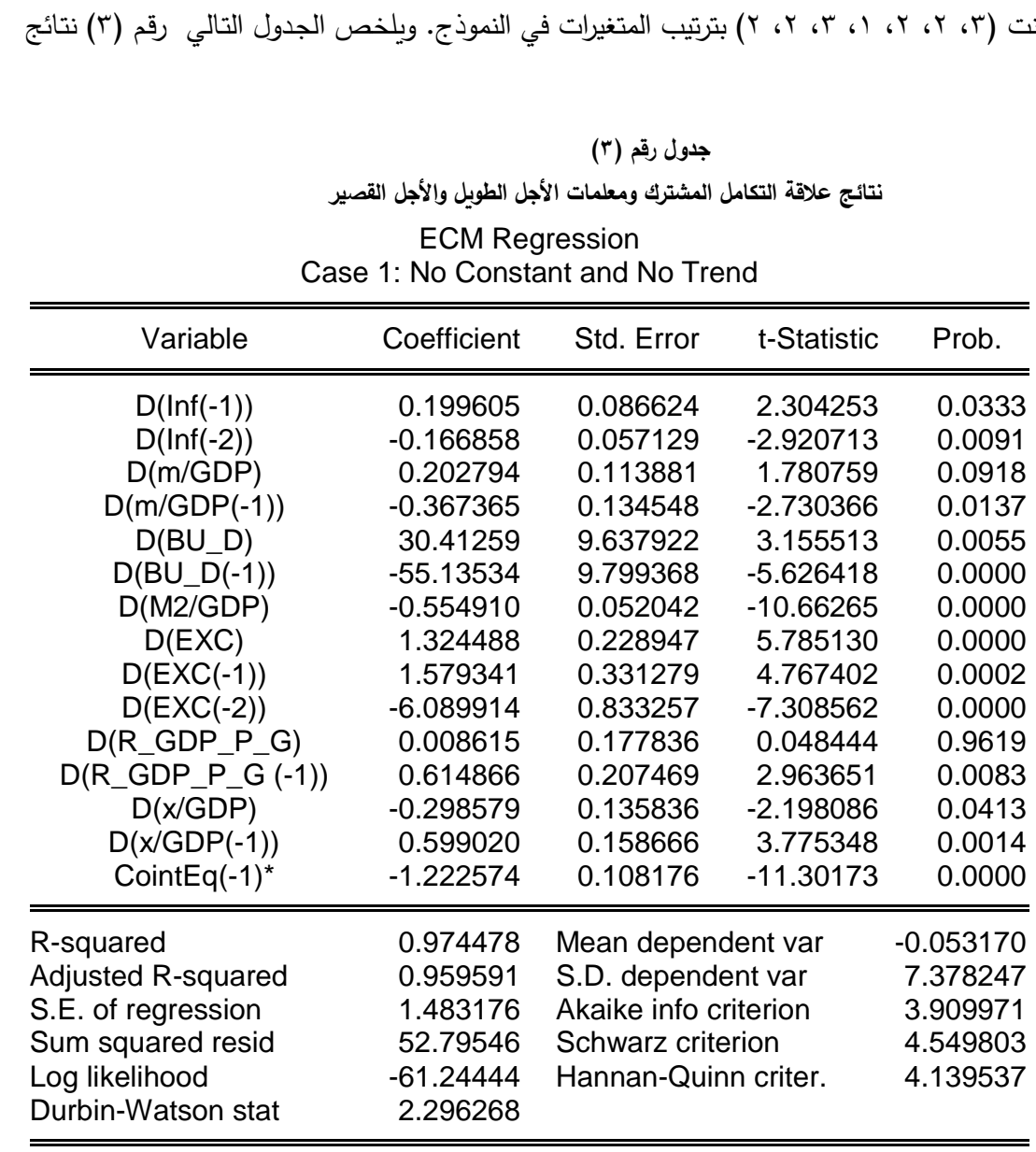

Levels Equation

Case 1: No Constant and No Trend

\begin{tabular}{ccccc}
\hline \hline Variable & Coefficient & Std. Error & t-Statistic & Prob. \\
\hline \hline m/GDP & 0.405933 & 0.149485 & 2.715546 & 0.0142 \\
BU_D & 44.86051 & 7.836607 & 5.724481 & 0.0000 \\
M2/GDP & -0.001725 & 0.024442 & -0.070574 & 0.9445 \\
EXC & 0.210903 & 0.202444 & 1.041784 & 0.3113 \\
R_GDP_P_G & -1.573522 & 0.228858 & -6.875549 & 0.0000 \\
x/GDP & -0.079441 & 0.138071 & -0.575365 & 0.5722 \\
\hline \hline
\end{tabular}

$\mathrm{EC}=\operatorname{lnf}-\left(0.4059^{\star} \mathrm{m} / \mathrm{GDP}+44.8605^{\star} \mathrm{BU} \_\mathrm{D}\right.$

$-0.0017^{\star} \mathrm{M} 2 / \mathrm{GDP}+0.2109^{\star} \mathrm{EXC}-1.5735^{\star} \mathrm{R} \_\mathrm{GDP} P$ P G $-0.0794^{\star} \mathrm{x} / \mathrm{GDP}$ ) 
و من الجدول السابق يلاحظ أن الانفتاح على الواردات m/GDP يرتبط ارتباط معنوي موجب بمعدل التضخم في الأجل القصير عند مستوى معنوية • (\%)، وأيضاً في الأجل الطويل و لكن عند مستوى معنوية أقل من ا\%٪. و هو ما يعني ان بيانات هذا النموذج تدعم رفض تحقق فرضية Romer في الاقتصاد المصري خلال فترة الدراسة. و يتفق ذلك مـع دراسـة ElHodaiby \& ElSamman El El Eلى الاقتصـاد المصري ، بالرغم من اختلاف المتغيرات المستخدمة و اختلاف فترة التقدير و طبيعة البيانات. كما يلاحظ أن العلاقة طردية بين العجز في الموازنة العامة للدولة و معدل التضخم عند مستوي المعنوية أقل من ا\% في كل من الأجلين القصير و الطويل. و بينما كانت معاملات عرض النقود الي الناتج و نسبة الصادرات الي الناتج سالبة و معنويـة في الأجل القصير إلا انها كانت غير معنويـة في الأجل الطويل . و يحدث العكس بالنسبة لمعامل معدل نمو نصيب الفرد من الناتج المحلي الاجمالي الحقيقي حيث كان سالب و معنوي في الاجل الطويل و غير معنوي في الاجل القصير •

أما سعر الصرف فيظهر ارتباطه الموجب بمعدل التضخم معنويا في الاجل القصير ، في حين تكون العلاقة غير معنوية في الاجل الطويل. وبالنظر إلى قيمة معامل تصحيح الأخطاء The Error Correction Term

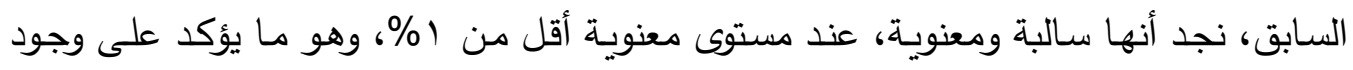

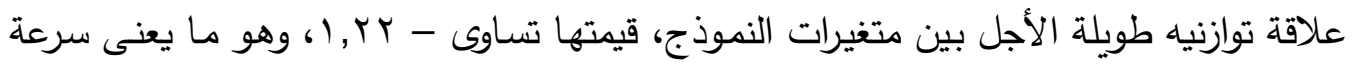

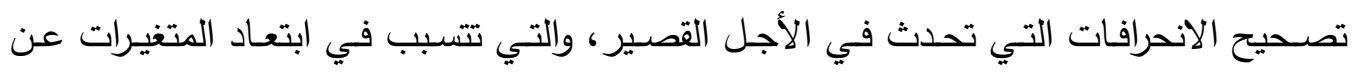


علاقتها التوازنيه طويلة الأجل، أي أن الانحراف يحدث في الأجل القصير في النموذج يستغرق

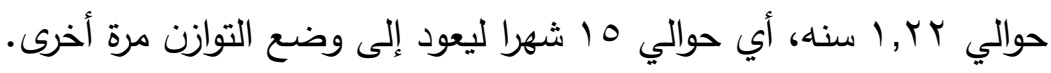

؟ - ـ - ب: "نموذج المؤشر التقليدي للانفتاح "

4- ـ - - 1 : اختبار التكامل المشترك

يعرض الجدول التالي رقم (ع) ملخصا لاختبار التكامل المشترك بين المتغيرات محل

الدراسة باستخدام اختبار الحدود للتكامل المشترك Bound Testing for Cointegration كما يلي:

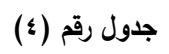

Bound Testing for Cointegration نتائج اختبار التكامل المشترك رف

F-Bounds Test Null Hypothesis: No levels relationship

\begin{tabular}{lcrrr}
\hline \hline Test Statistic & Value & Signif. & $\mathrm{I}(0)$ & $\mathrm{I}(1)$ \\
\hline \hline F-statistic & 23.36288 & $10 \%$ & 2.26 & 3.35 \\
$\mathrm{k}$ & 5 & $5 \%$ & 2.62 & 3.79 \\
& & $2.5 \%$ & 2.96 & 4.18 \\
& & $1 \%$ & 3.41 & 4.68 \\
\hline \hline
\end{tabular}

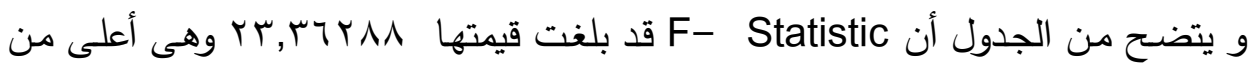
القيمة الجدولية الحرجة ، للحد الأعلى، عند مستوى معنوية ا \%. وبالتالي يُمكن رفض فرض العدم، الذى ينص على عدم وجود علاقة طويلة الأجل بين متغيرات النموذج. أي أنه يوحد علاقة تكامل مُشترك Cointegration طويلة الأجل بين المتغيرات محل الدراسة. ؟ - ؟ - - - : تقدير معلمات النموذج

وهنا أيضا، قبل استخدام أسلوب الانحدار الذاتي لفترات الابطاء الموزعـة ARDL لتقدير معلمات النموذج تم تحديد العدد الأمثل لفترات الابطاء اللازمـة لكل متغير باستخدام معيار 


\begin{tabular}{|c|c|c|c|c|}
\hline \multicolumn{5}{|c|}{ 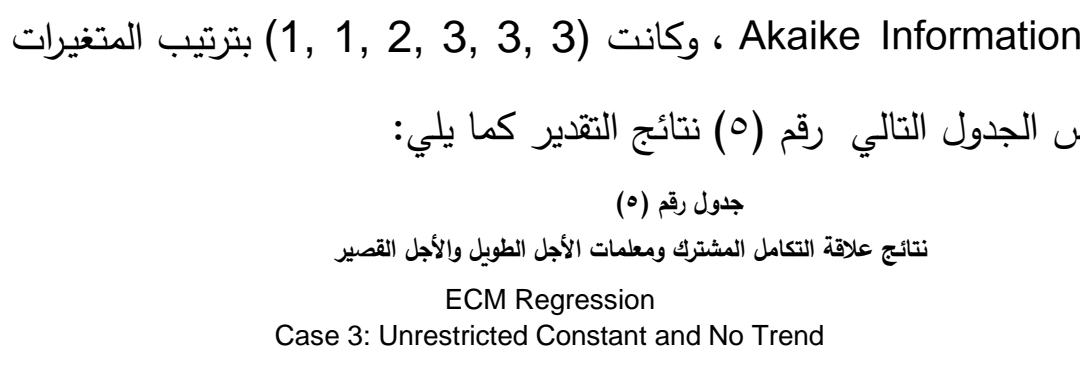 } \\
\hline Variable & Coefficient & Std. Error & t-Statistic & Prob. \\
\hline C & 13.96793 & 1.091451 & 12.79757 & 0.0000 \\
\hline $\mathrm{D}((\mathrm{x}+\mathrm{m}) / \mathrm{GDP})$ & 0.188317 & 0.056570 & 3.328953 & 0.0033 \\
\hline D(BU_D) & 11.99414 & 9.395207 & 1.276623 & 0.2164 \\
\hline $\mathrm{D}\left(\mathrm{BU} \_\overline{\mathrm{D}}(-1)\right)$ & -72.44260 & 10.29397 & -7.037383 & 0.0000 \\
\hline $\mathrm{D}(\mathrm{M} 2 / \mathrm{GDP})$ & -0.552711 & 0.052222 & -10.58377 & 0.0000 \\
\hline $\mathrm{D}(\mathrm{M} 2 / \mathrm{GDP}(-1))$ & -0.360212 & 0.101841 & -3.537002 & 0.0021 \\
\hline $\mathrm{D}(\mathrm{M} 2 / \mathrm{GDP}(-2))$ & 0.489038 & 0.066810 & 7.319877 & 0.0000 \\
\hline $\mathrm{D}(\mathrm{EXC})$ & 2.103839 & 0.297660 & 7.067938 & 0.0000 \\
\hline $\mathrm{D}(\mathrm{EXC}(-1))$ & 1.578535 & 0.379501 & 4.159496 & 0.0005 \\
\hline $\mathrm{D}(\mathrm{EXC}(-2))$ & -7.735725 & 0.990001 & -7.813858 & 0.0000 \\
\hline$D\left(R \_G D P\right.$ P_G $)$ & -0.452784 & 0.186728 & -2.424832 & 0.0249 \\
\hline$D\left(R \_G D P\right.$ P_G $\left.(-1)\right)$ & 0.656496 & 0.187116 & 3.508495 & 0.0022 \\
\hline$D(R$ GDP_P_G (-2)) & 0.198255 & 0.169206 & 1.171679 & 0.2551 \\
\hline CointEq $\overline{(-1)^{*}}$ & -1.302604 & 0.098405 & -13.23713 & 0.0000 \\
\hline R-squared & 0.967096 & \multicolumn{2}{|c|}{ Mean dependent var } & -0.053170 \\
\hline Adjusted R-squared & 0.949986 & \multicolumn{2}{|c|}{ S.D. dependent var } & 7.378247 \\
\hline S.E. of regression & 1.650050 & \multicolumn{2}{|c|}{ Akaike info criterion } & 4.112751 \\
\hline Sum squared resid & 68.06662 & \multicolumn{2}{|c|}{ Schwarz criterion } & 4.709927 \\
\hline Log likelihood & -66.19865 & \multicolumn{2}{|c|}{ Hannan-Quinn criter. } & 4.327013 \\
\hline F-statistic & 56.52260 & \multicolumn{2}{|c|}{ Durbin-Watson stat } & 2.080667 \\
\hline Prob(F-statistic) & 0.000000 & & & \\
\hline
\end{tabular}

Levels Equation

Case 3: Unrestricted Constant and No Trend

\begin{tabular}{crrrr}
\hline \hline Variable & Coefficient & Std. Error & t-Statistic & Prob. \\
\hline \hline (x+m)/GDP & 0.205537 & 0.030964 & 6.638011 & 0.0000 \\
BU_D & 47.97421 & 4.961282 & 9.669719 & 0.0000 \\
M2/GDP & -0.128561 & 0.042365 & -3.034633 & 0.0065 \\
EXC & 0.148508 & 0.187761 & 0.790944 & 0.4383 \\
R_GDP_P_G & -1.779894 & 0.269478 & -6.604972 & 0.0000 \\
\hline \hline
\end{tabular}

$E C=\operatorname{lnf}-\left(0.2055^{\star}\left((x+m) / G D P+47.9742^{*} B U \_D-0.1286^{\star} M 2 / G D P+0.1485\right.\right.$

${ }^{\star} E X C$-1.7799* R_GDP_P_G) 
ومن الجدول السابق يلاحظ أن المؤشر التقليدي للانفتاح x+ m) /GDP يرتبط ارتباط

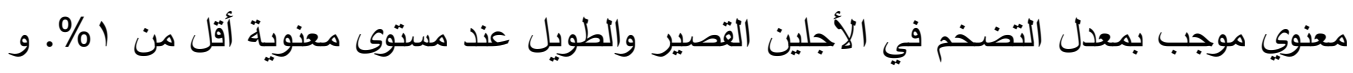

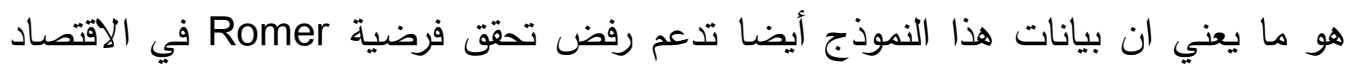

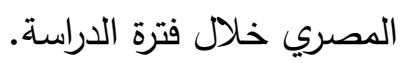

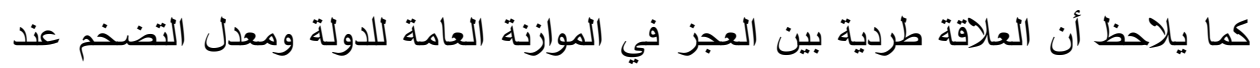
مستوي المعنوية أقل من (\% في كل من الأجل الطويل فقط وغير معنوية في الاجل القصير. و بينما كانت معاملات عرض النقود الي الناتج و معدل نمو نصيب الفرد من الناتج

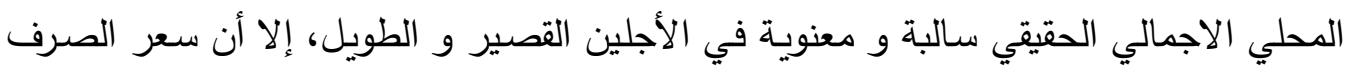

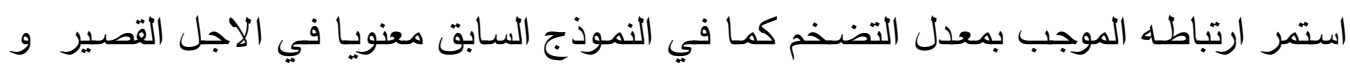
غير معنوي في الاجل الطويل. وبالنظر إلى قيمة معامل تصحيح الأخطاء $\varphi$ The Error Correction Term في الجدول

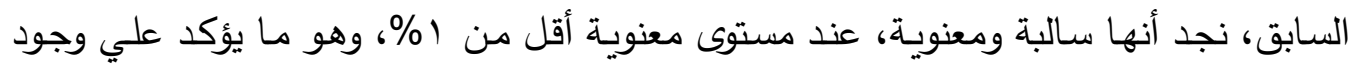

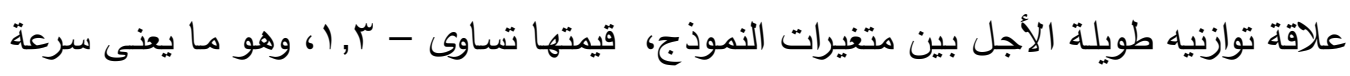

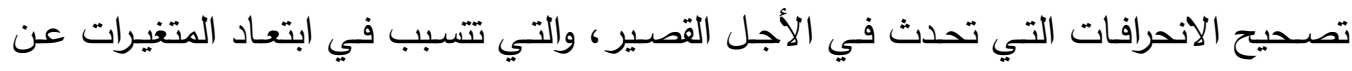

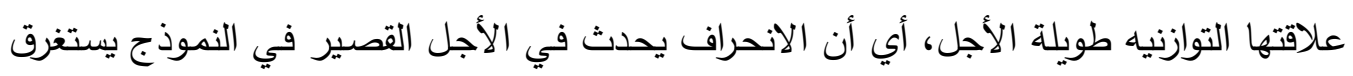

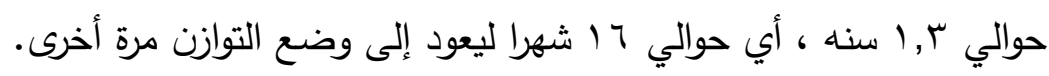

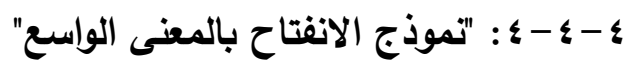
4- צ - צ - 1: اختبار التكامل المشترك

وتتلخص نتائج هذا الاختبار في الجدول التالي رقم (؟). 


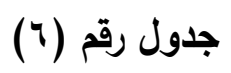

Bound Testing for Cointegration نتائج اختبار التكامل المشترك

\begin{tabular}{lcccc} 
F-Bounds Test & & \multicolumn{2}{c}{$\begin{array}{r}\text { Null Hypothesis: No levels } \\
\text { relationship }\end{array}$} \\
\hline \hline Test Statistic & Value & Signif. & $\mathrm{I}(0)$ & $\mathrm{I}(1)$ \\
\hline \hline F-statistic & \multirow{2}{*}{$\mathrm{T}$ k } & $10 \%$ & 1.81 & 2.93 \\
k & 5 & $5 \%$ & 2.14 & 3.34 \\
& & $2.5 \%$ & 2.44 & 3.71 \\
& & $1 \%$ & 2.82 & 4.21 \\
\hline
\end{tabular}

و يتضح من الجدول أن F- Statistic قد بلغت قيمتها 7.770869 وهى أعلى من القيهة الجدولية الحرجة ، للحد الأعلى، عند مستوى معنويـة ا\%. وبالتالي يُمكن رفض فرض العدم، الذى ينص على عدم وجود علاقة طويلة الأجل بين متغيرات النـوذج. أي أنه يوحد علاقة تكامل مُشترك Cointegration، طويلة الأجل، بين المتغيرات محل الدراسة.

$$
\text { צ - צ - צ - ب: تقدير معلمات النموذج }
$$

و كذلك في هذا النموذج، تم في البداية تحديد العدد الأمثل لفترات الابطاء اللازمة لكل

متغير باستخدام معيار (Akaike Information Criteria (AIC) ، وكانت , 3, 1, 4, 3, 3) (1 بترتيب المتغيرات في النموذج. ثم استخدم أسلوب الانحدار الذاتي لفترات الابطاء الموزعة ARDL لتددير معلمات النموذج ، ويعرض الجدول التالي رقم (V) نتائج هذا التقدير كما يلي: 


\section{جدول رقم (v)}

نتائج علاقة التكامل المشترك ومعلمات الأجل الطويل والأجل القصير

ECM Regression

Case 1: No Constant and No Trend

\begin{tabular}{crrrr}
\hline \hline Variable & Coefficient & Std. Error & t-Statistic & Prob. \\
\hline \hline D(Inf(-1)) & -0.494779 & 0.079429 & -6.229162 & 0.0000 \\
D(Inf(-2)) & -0.189712 & 0.059513 & -3.187731 & 0.0051 \\
D(KOF) & 0.026026 & 0.050887 & 0.511443 & 0.6153 \\
D(BU_D) & 2.118682 & 10.72580 & 0.197531 & 0.8456 \\
D(BU_D(-1)) & -15.30000 & 10.61883 & -1.440837 & 0.1668 \\
D(BU_D(-2)) & 55.51008 & 10.91875 & 5.083921 & 0.0001 \\
D(BU_D(-3)) & 18.91015 & 10.38433 & 1.821028 & 0.0853 \\
D(M2/GDP) & -0.500237 & 0.059828 & -8.361326 & 0.0000 \\
D(M2/GDP(-1)) & -0.084795 & 0.114757 & -0.738910 & 0.4695 \\
D(M2/GDP(-2)) & 0.688002 & 0.123967 & 5.549868 & 0.0000 \\
D(EXC) & 1.197412 & 0.339091 & 3.531245 & 0.0024 \\
D(EXC(-1)) & 0.865909 & 0.388182 & 2.230678 & 0.0387 \\
D(EXC(-2)) & -8.339790 & 1.029973 & -8.097092 & 0.0000 \\
D(R_GDP_P_G) & -0.685045 & 0.248166 & -2.760425 & 0.0129 \\
CointEq(-1) & -0.349751 & 0.045313 & -7.718592 & 0.0000 \\
\hline \hline R-squared & 0.965174 & Mean dependent var & 0.236720 \\
Adjusted R-squared & 0.943975 & S.D. dependent var & 7.248713 \\
S.E. of regression & 1.715738 & Akaike info criterion & 4.204946 \\
Sum squared resid & 67.70643 & Schwarz criterion & 4.851361 \\
Log likelihood & -64.89397 & Hannan-Quinn criter. & 4.434935 \\
Durbin-Watson stat & 2.158804 & & & \\
\hline \hline
\end{tabular}

Levels Equation

Case 1: No Constant and No Trend

\begin{tabular}{crrrr}
\hline \hline Variable & Coefficient & Std. Error & t-Statistic & Prob. \\
\hline \hline KOF & -0.168413 & 0.233566 & -0.721052 & 0.4801 \\
BU_D & 0.124773 & 61.65148 & 0.002024 & 0.9984 \\
M2/GDP & 0.442811 & 0.349331 & 1.267596 & 0.2211 \\
EXC & -0.241355 & 1.231875 & -0.195925 & 0.8469 \\
R_GDP_P_G & -4.587337 & 2.718858 & -1.687229 & 0.1088 \\
\hline \hline EC = INF_DEF - $\left(-0.1684^{*}\right.$ KOF + 0.1248*BU_D + 0.4428*M2/GDP & \\
-0.2414*EXC $-4.5873^{*}$ R_GDP_P_G ) & &
\end{tabular}


و من الجدول السابق يلاحظ أن العلاقة بين مؤشر العولمة الاقتصادية KOF و معدل

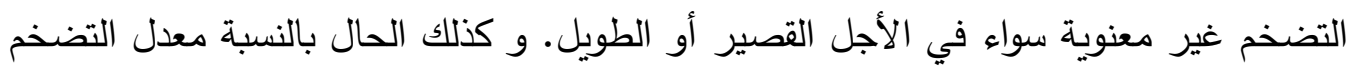
مع العزز في الموازنة العامة للدولة و نسبة عرض الاجل النقود الي الناتج. و بينما تحول معامل معدل نمو نصيب الفرد من الناتج المحلي الاجمالي الحقيقي السالب

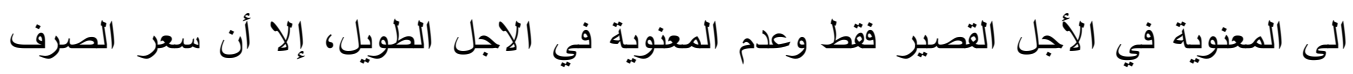

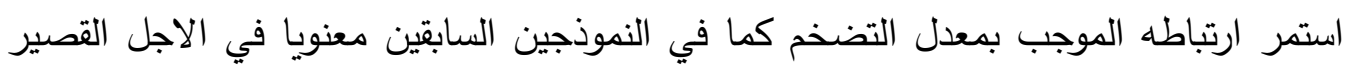
و غير معنوي في الاجل الطويل.

وحيث أن معامل الانفتاح و معظم معاملات النموذج كانت غير معنوية فإنه لا يمكن

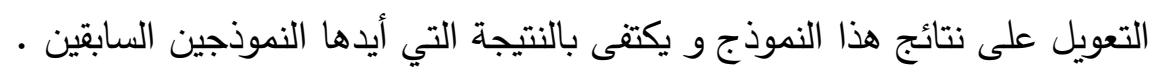




\section{๑ : النتائج و التوصيات}

-كثفت النتائج العملية عدم تحقق فرضية Romer (1993) بالنسبة للاقتصـاد المصري و هو ما تمثل في اكتشاف أن العلاقة طردية بين الانفتاح التجاري و معدل التضخم ـ أي أن الزيادة في الانفتاح التجاري من المتوقع أن يترتب عليها ارتفاع في معدلات التضـخم في الاقتصـاد المصري • و يمكن ارجاع ذلك إلي اعتماد الاقتصاد المصري على الواردات في تغطية جزء من الاحتياجات الضرورية من السلع الغذائية و مستلزمات الانتاج ، و هو ما يعني انعكاس ارتفاع

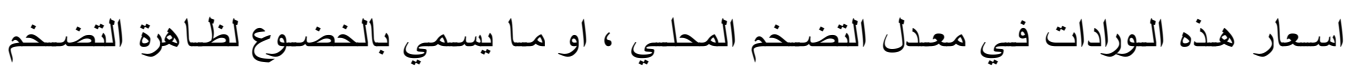

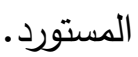

و يترتب علي ذلك توصية هامسة جدا خاصـة بالسياسـة الاقتصـادية بتوخي الحذر في اتخـاذ القرارات التي تزيد من درجة الانفتاح التجاري و دراسـة ما قد ينجم عنها من تأثير علي معدل • التضخم

- كما اشارت النتائج ايضا الى وجود علاقة معنوية موجبة بين العجز المالي و معدل التضخم، و يتوافق ذلك مـع ما سبق ذكره من الاثر التوسعي المتوقع للعجز المالي حتي و ان تم تمويله من مصادر حقيقية.

و يدعو ذلك الى التوصية بضـرورة الالتزام بحد أقصسي لنسبة العجز المـالي الى النـاتج المحلي الاجمالي حتي لا يزيد من الضغوط التضخمية الحالية او المتوقعة في الاقتصاد.

- و كذلك الحسال بالنسبة للعلاقة المعنويـة الموجبـة بين سعر صـرف العملـة الاجنبيـة و معدل التضــخم في الاجـل القصـير ، و التي تعنـي ان تخفيض سـعر الصـرف يترتب عليـه ارتفــاع مستويات الاسعار في الاجل القصير و هو ما يدعم فكرة التضخم المستورد و الذي ظهر اثره مع الانخفاضات التي حدثت في سعر صرف الجنيه المصري عام 1 ـ ـ على اعتبار ان مصر مستورد صافي. 
و بناء علي ذلك يمكن التوصية بتجنب التخفيض الكبير في قيمة العملة المحلية لما يحمله في طياته من ضغوط تطخمية ، بالاضافة لما يترتب عليه من تفاقم في ازمة الدديونية. - أما العلاقة المعنويـة السالبة بين معدل النمو في نصيب الفرد من الناتج المحلي الاجمالي و

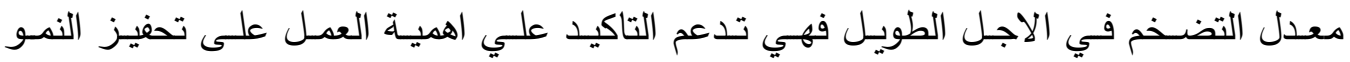

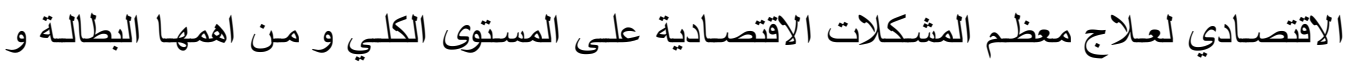
التضخم.

مقترحات لبحوث مستقبلية: من الموضوعات المرتبطة بمجال البحث و التي قد يكون من المفيد التركيز عليها بشيء من التفصيل في بحوث مستقبلية على الاقتصاد المصري: الانفتاح المالي و اثره علي التضخم و النمو الاقتصادي، و الانفتاح التجاري و درجة انحدار منحنى فيليبس. 
1. Ashra, S. (2002). Inflation and openness: a study of selected developing economies. Indian Council for Research on International Economic Relations. Working Paper, (84).

2. Binici, M., Cheung, Y. W., \& Lai, K. S. (2012). Trade openness, market competition, and inflation: Some sectoral evidence from OECD countries. International Journal of Finance \& Economics, 17(4), 321336.

3. Chhabra .M and Alam. Q, (2020) "An empirical study of trade openness and inflation in India", Decision, 47(1):79-90

4. Dickey, D. A. and W. A. Fuller (1979). "Distribution of the Estimators for Autoregressive Time Series with a Unit Root." Journal of the American Statistical Association 74(366): 427-431.

5. ElHodaiby, M. W. \& ElSamman, A. (2021). The Relation between Central Bank Independence and Inflation Rate in Egypt: An Empirical Analysis during 1998-2019, International Journal of Economics and Financial Issues, 2021, 11(1), 114-125.

6. Evans, R. W. (2007). Is openness inflationary? imperfect competition and monetary market power. Globalization and Monetary Policy Institute, Federal Reserve Bank of Dallas, GMPI Working Paper, (1).

7. Galal, S. \& Lan, D.Y. (2017). Relationship between Inflation and Foreign Trade, International Journal of Business Marketing and Management, 2(5): 1-7.

8. Gokal, V., \& Hanif, S. (2004). Relationship between inflation and economic growth. Economics Department, Reserve Bank of Fiji. 
9. Granato, J., Lo, M., and Wong, M. C. Sunny (2007). “A note on Romer's openness-inflation relation: the responsiveness of AS and AD to economic openness and monetary policy." Applied Economics 39, 191197.

10. Israr,M.F.K., (2017) "Trade Openness and Inflation in Pakistan: A Cointegration and Causality Analysis (1973-2015)"Master thesis in economics, UNIVERSITY OF ÉVORA.

file:///C:/Users/Dr\%20Khaled\%20Ibrahim/Downloads/Documents/Maste rs\%20in\%20Economics_2.pdf

11. Jedidia, K. B., Dammak, T. B., \& Kamel, H. (2019). Trade-threshold Effect on Inflation in Tunisia: New Evidence Resulting from a Nonlinear Approach. International Economic Journal, 33(1), 149-169.

12. Joshi, A. R., \& Acharya, D. (2010). Inflation and trade openness: Empirical investigation for India. IUP Journal of Monetary Economics, 8(1/2), 113.

13. Kim, M., Beladi, H., (2005). Is free trade deflationary. Economic Letters, 89: 343-49.

14. Lidiema ‘C. (2020).” Trade Openness and Crude Oil Price Effects on Food Inflation: Examining the Romer Hypothesis in Kenya". Journal of World Economic Research. Vol. 9, No. 2, pp. 91-100.

15. Lin, H.Y. (2010). Openness and Inflation Revisited. International Research Journal of Finance and Economics, 37, 40-45.

16. Lin, F., Mei, D., Wang, H., and Yao, X. (2017). Romer was right on openness and inflation: evidence from Sub-Saharan Africa. Journal of Applied Economics, 20(1):121-140.

17. Lotfalipour, M. R., Montazeri, S., \& Sedighi, S. (2013). Trade Openness and Inflation. Evidence from MENA Region 
Countries. Petroleum-Gas University of Ploiesti Bulletin, Technical Series, 65(2).

18. Mansilla, F. M., Arruda, E. F., and Ferreira, R. T., (2020) "Trade openness and inflation dynamics in Brazil", Economics Bulletin, 40(3), pp 1948-1957 21.

19. Marinheiro, C. F. (2004). Fiscal sustainability of Egypt's public finances. FEMISE working paper (March).

20. Mukhtar, T., Jehan, Z., \& Bilquees, F. (2019). Is trade openness inflationary in developing economies: an asymmetric analysis for Pakistan. Pakistan Economic and Social Review, 57(1), 47.

21. Pesaran, M. H., Shin, Y., \& Smith, R. J. (2001). Bounds testing approaches to the analysis of level relationships. Journal of applied econometrics, 16(3), 289-326.

22. Romer, D. (1993). Openness and inflation: theory and evidence. The quarterly journal of economics, 108(4), 869-903.

23.Sahu, P., \& Sharma, N.K. (2018). Impact of Trade Openness on Inflation in India: An Autoregressive Distributed Lag (ARDL) Approach. The Empirical Economics Letters, 17(1), 21 - 32

24. Samimi, A. J., Ghaderi, S., Hosseinzadeh, R., \& Nademi, Y. (2012). Openness and inflation: New empirical panel data evidence. Economics Letters, 117(3), 573-577.

25. Sikdar, A., Kundu, N., Khan, Z. S. (2013). Trade openness and inflation: A test of Romer hypothesis for Bangladesh MPRA Paper No. 65244, University Library of Munich, Germany.

26. Terra, C. T. (1998). Openness and inflation: a new assessment. The Quarterly Journal of Economics, 113(2), 641-648. 
27. Thomas, C. (2012). Trade openness and inflation: Panel data evidence for the Caribbean. International Business \& Economics Research Journal (IBER), 11(5), 507-516.

28. Ul Haq, I., Alotaish, M. S. M., Kumara, N. G. S., and Otamurodov, S. (2014). Revisiting the Romer's hypothesis: Time series evidence from small open economy, Pakistan Journal of Applied Economics 24 (1), 115.

29. ul Haq, I., Zhu, S., Shafiq, M., \& Khan, A. (2016). Does Proxy of Openness or Methodology Matter to Hold Romer's Hypothesis? International Journal of Economics, Commerce and Management, 4(1): $1-12$.

30. Wahu, M. C. (2016). Relationship between Openness and Inflation in Kenya: Testing Romer Hypothesis using Autoregressive Distributive Lag. (B.Sc. Project, Strathmore University, Strathmore Institute of Mathematical Sciences, Strathmore University Nairobi, Kenya).

31. World Bank, (2020). World Development Report. Oxford: Oxford University Press.

32. Zakaria, M. (2010), Openness and inflation: Evidence from time series data. Dogus Universitesi Dergisi, Volume 11(2), pp. 313-322.

33. World Development Indicators | DataBank (worldbank.org)

34. http://www.mof.gov.eg 